\title{
Semiclassical Trace Formula and Spectral Shift Function for Systems via a Stationary Approach
}

\author{
MAROUANE ASSAL, MOUEZ DIMASSI AND SETSURO FUJIIÉ
}

\begin{abstract}
We establish a semiclassical trace formula in a general framework of microhyperbolic hermitian systems of $h$-pseudodifferential operators, and apply it to the study of the spectral shift function associated to a pair of selfadjoint Schrödinger operators with matrix-valued potentials. We give Weyl type semiclassical asymptotics with sharp remainder estimate for the spectral shift function, and, under the existence of a scalar escape function, a full asymptotic expansion in the strong sense for its derivative. A time-independent approach enables us to treat certain potentials with energy-level crossings.
\end{abstract}

\section{INTRODUCTION}

In this paper, we study the spectral shift function (SSF for short) for Schrödinger operators with matrix-valued potentials. Such operators appear in molecular physics in the BornOppenheimer approximation. The justification of this approximation and a classification of matrix Schrödinger operators can be found in [5, 10, 15, 17].

More precisely, we are concerned with the SSF for the pair of operators $\left(P_{1}, P_{0}\right)$ with

$$
P_{0}:=-h^{2} \Delta \otimes I_{N}+V_{\infty}, \quad P_{1}:=-h^{2} \Delta \otimes I_{N}+V(x),
$$

where $h \in(0,1]$ is a small positive parameter, $I_{N}$ is the identity $N \times N$ matrix, $V_{\infty}$ is an $N \times N$ constant hermitian matrix and $V(x)$ is a smooth hermitian matrix-valued potential which tends rapidly enough to $V_{\infty}$ at infinity. The SSF associated to $\left(P_{1}, P_{0}\right)$ denoted $s_{h}$ is defined as distribution (modulo a constant) by the Lifshits-Krein formula

$$
\left\langle s_{h}^{\prime}, f\right\rangle=-\operatorname{tr}\left(f\left(P_{1}\right)-f\left(P_{0}\right)\right), \quad \forall f \in C_{0}^{\infty}(\mathbb{R} ; \mathbb{R}) .
$$

The SSF is related with the eigenvalue counting function below the level inf $\sigma\left(P_{0}\right)$ and with the scattering determinant above this level (Birman-Krein formula, see [36]). Here $\sigma\left(P_{0}\right)$ stands for the spectrum of $P_{0}$.

The concept of the SSF was introduced in the middle of the previous century by I. M. Lifshits in his investigations in the solid state theory (see [22, 23]) and then developed by M. Krein (see [20, 21, 19]) into a mathematical theory. The work of Krein on the SSF has been described in details in the survey [3]. One can also find detailed account concerning mathematical and historical aspects of the SSF in [2].

In the scalar case $N=1$, a lot of works have been devoted to the study of the SSF in different asymptotic regimes (see [28] and the references therein). In particular, a Weyl-type asymptotics of the SSF with a sharp remainder estimate and a complete asymptotic expansion

2010 Mathematics Subject Classification. 81Q10 (47A55, 81Q20, 47N50).

Key words and phrases. Spectral shift function, matrix Schrödinger operators, asymptotic expansions. 
of the derivative of the SSF were studied in high energy regime ([29]) and in the semiclassical regime (30], 31]).

The proofs of these works reduce to the study of

$$
\operatorname{tr}\left(f\left(P_{1}\right) \mathcal{F}_{h}^{-1} \theta\left(\tau-P_{1}\right)-f\left(P_{0}\right) \mathcal{F}_{h}^{-1} \theta\left(\tau-P_{0}\right)\right),
$$

where $\theta$ is a smooth function of the time $t$ with compact support and $\mathcal{F}_{h}^{-1}$ is the semiclassical Fourier inverse transform defined by (2.2). The method in [30 consists in writing (1.3) as the semiclassical Fourier inverse transform of $\theta(t) \operatorname{tr}\left(f\left(P_{1}\right) e^{-i t P_{1} / h}-f\left(P_{0}\right) e^{-i t P_{0} / h}\right)$, and constructing (modulo $\mathcal{O}\left(h^{\infty}\right)$ ) the Schwartz' kernel of the evolution operator $f\left(P_{1}\right) e^{-i t P_{1} / h}$. This construction by means of Fourier integral operators is now standard and well known for scalar-valued operators $P_{1}$ (see [12, 14] for problems concerning the asymptotic distribution of eigenvalues, and [28, 29, 30] for the SSF). For matrix-valued operators this explicit construction is very complicated (or impossible). To avoid this problem, and to study the counting function of eigenvalues of $P_{1}, \mathrm{~V}$. Ivrii [14] observed that a rough construction by using the successive approximation method of $f\left(P_{1}\right) e^{-i t P_{1} / h}$ for $|t|<h^{1-\delta}$ (with $0<\delta \leq 1$ ) suffices to get a full asymptotic expansion in powers of $h$ of $\operatorname{tr}\left(f\left(P_{1}\right) \mathcal{F}_{h}^{-1} \theta\left(\tau-P_{1}\right)\right)$. This beautiful observation is used by the second author and J. Sjöstrand [7] to develop a time-independent approach to get asymptotics of $\operatorname{tr}\left(f\left(P_{1}\right) \mathcal{F}_{h}^{-1} \theta\left(\tau-P_{1}\right)\right)$ for matrix-valued operator $P_{1}$. The novelty in this approach consists in expressing (1.3) in terms of the resolvent instead of evolution operator, and studying the (almost) analyticity of its trace near the real axis. This method is used in [8] to study the SSF for scalar non semi-bounded operators such as Stark Hamiltonian. The aim of this paper is to develop and apply this stationary approach to the study of the SSF for matrix-valued operators.

In the first part of this work, we consider a general system of $h$-pseudodifferential operator $H^{w}=H^{w}\left(x, h D_{x}\right)$. For a fixed energy $\tau_{0}$ such that $\tau_{0}-H(x, \xi)$ is uniformly microhyperbolic in some direction $T$ (see Definition 2.1), we show that the trace of the operator $\chi^{w} f\left(H^{w}\right) \mathcal{F}_{h}^{-1} \theta\left(\tau-H^{w}\right)$ is negligible $\left(=\mathcal{O}\left(h^{\infty}\right)\right)$ provided that $\theta$ is supported in $h^{1-\delta} \leq|t| \leq \kappa$ (for arbitrary positive $h$-independent $\kappa$ ), see Theorem 2.2 . Here $\chi \in C_{0}^{\infty}\left(\mathbb{R}^{2 n} ; \mathbb{R}\right.$ ) and $f$ is supported in a small neighborhood of $\tau_{0}$. Moreover, under the existence of an escape function associate to $H(x, \xi)$ at $\tau_{0}$ (see (2.15)), we can take $\kappa=h^{-\nu}$ for arbitrary $\nu>0$ (see Remark 3.1 and section 4.4). On the other hand, we give a complete asymptotic expansion in powers of $h$ of $\operatorname{tr}\left(\chi^{w} f\left(H^{w}\right) \mathcal{F}_{h}^{-1} \theta\left(\tau-H^{w}\right)\right)$ provided that $\theta$ is supported in a small $h$-independent neighborhood of 0 and $\tau_{0}-H(x, \xi)$ is microhyperbolic at every point $(x, \xi) \in \operatorname{supp} \chi$, see Theorem 2.4. This is a consequence from the fact that the above trace depends, modulo $\mathcal{O}\left(h^{\infty}\right)$, only on the symbol $\tau_{0}-H(x, \xi)$ on the support of $\chi$ as long as the support of $\theta$ is small enough near 0 (Theorem 2.3), and the fact that a symbol $\tau_{0}-H$ microhyperbolic near a point can be extended to a uniformly microhyperbolic symbol in the whole phase space (Theorem A.3).

To our best knowledge, there are only few works treating the semiclassical asymptotics of the SSF for matrix valued operators (see [4, 18, and the references therein). The asymptotics of the SSF for the semi-classical Dirac operator has been studied in [4. In this case, the classical corresponding Hamiltonian has uniformly distinct eigenvalues, and then the study of the SSF can be reduced to the scalar case by diagonalization. The relation between the spectral shift function and the resonances for Dirac operator with analytic potential has been examined in [18]. In the second part of this paper, we consider the SSF associated to the pair 
of Schrödinger operators with matrix-valued potentials defined in (1.1), without any condition on the multiplicities of its eigenvalues. First, using Theorem 2.4, we show that (1.3) has a full asymptotic expansion in $h$ when the support of $\theta$ is close enough to the origin (Theorem 2.6). This result with a Tauberian argument give the Weyl-type asymptotic formula for the SSF with a sharp remainder estimate (Theorem 2.7). Finally we give a pointwise full asymptotic expansion of the derivative of the SSF near energies $\tau$ where there exists a scalar escape function associated to the classical Hamiltonian $\xi^{2} I_{N}+V(x)$ (Theorem 2.8). This last theorem is a generalization to the matrix case of the result of [30] at non-trapping energies.

The paper is organised as follows. In section 2, we state our main results and we give an outline of the proofs. The proofs of these results will be given in Sections 3 and 4 respectively. Finally, the appendix A contains some technical lemmas related to the notion of microhyperbolicity used in our proofs.

Notations : For $\xi=\left(\xi_{1}, \ldots, \xi_{n}\right) \in \mathbb{R}^{n}$, we use the usual notation $\langle\xi\rangle:=\left(1+\xi^{2}\right)^{1 / 2}$, where $\xi^{2}:=\xi_{1}^{2}+\ldots+\xi_{n}^{2}=|\xi|^{2}$. For $z \in \mathbb{C}$, we recall that $\bar{\partial}_{z}:=\frac{1}{2}\left(\partial_{\Re z}+i \partial_{\Im z}\right)$. The bracket $\left[a_{j}\right]_{0}^{1}$ stands for the difference $a_{1}-a_{0}$. The scalar products in $\mathbb{R}^{n}$ and $\mathbb{C}^{N}$ will be denoted $\langle$,$\rangle and$ $($,$) respectively. We introduce the following standard asymptotic notations that we shall$ use through the paper. Given a function $f_{h}$ depending on a small parameter $h \in(0,1]$, the relation $f_{h}=\mathcal{O}\left(h^{\infty}\right)$ (or $f_{h} \equiv 0$ ) means that $f_{h}=\mathcal{O}\left(h^{k}\right)$, for all $k \in \mathbb{N}$ and $h$ small enough. We write $f_{h} \sim \sum_{j \geq 0} \gamma_{j} h^{j}$ provided that for each $k \in \mathbb{N}, f_{h}-\sum_{j=0}^{k} \gamma_{j} h^{j}=\mathcal{O}\left(h^{k+1}\right)$.

\section{Statement of the Results}

Let $\mathcal{H}_{N}$ be the space of hermitian $N \times N$ matrices endowed with the norm $\|\cdot\|_{N \times N}$, where for $A \in \mathcal{H}_{N},\|A\|_{N \times N}:=\sup _{\left\{w \in \mathbb{R}^{N} ;|w|<1\right\}}|A w|$.

Throughout this work we will use the notations of [7] for symbols and $h$-pseudodifferential operators (see also [14]). In particular, $S^{0}\left(\mathbb{R}^{2 n} ; \mathcal{H}_{N}\right)$ is the class of symbols

$$
S^{0}\left(\mathbb{R}^{2 n} ; \mathcal{H}_{N}\right):=\left\{H \in C^{\infty}\left(\mathbb{R}^{2 n} ; \mathcal{H}_{N}\right) ;\left\|\partial_{x}^{\alpha} \partial_{\xi}^{\beta} H(x, \xi)\right\|_{N \times N}=\mathcal{O}_{\alpha, \beta}(1), \forall \alpha, \beta\right\} .
$$

We use the standard Weyl quantization of symbols. More precisely, if $H \in S^{0}\left(\mathbb{R}^{2 n} ; \mathcal{H}_{N}\right)$ then $H^{w}\left(x, h D_{x}\right)$ is the operator defined by

$$
H^{w}\left(x, h D_{x}\right) u(x)=\frac{1}{(2 \pi h)^{n}} \iint_{\mathbb{R}^{2 n}} e^{i(x-y) \cdot \xi / h} H\left(\frac{x+y}{2}, \xi\right) u(y) d y d \xi, \quad u \in C_{0}^{\infty}\left(\mathbb{R}^{n} ; \mathbb{C}^{N}\right) .
$$

We will occasionally use the shorthand notations $\mathrm{Op}_{h}^{w}(H)=H^{w}=H^{w}\left(x, h D_{x}\right)$ when there is no ambiguity.

We recall the following notion of microhyperbolicity which will play an important role in this paper.

Definition 2.1 (Microhyperbolicity). Let $H \in C^{\infty}\left(\mathbb{R}^{2 n} ; \mathcal{H}_{N}\right)$. We say that $H(x, \xi)$ is microhyperbolic at $\left(x_{0}, \xi_{0}\right)$ in the direction $T \in \mathbb{R}^{2 n}$, if there are constants $C_{0}, C_{1}, C_{2}>0$ such that

$$
\left(\left\langle T, \nabla_{x, \xi} H(x, \xi)\right\rangle w, w\right) \geq C_{0}|w|^{2}-C_{1}|H(x, \xi) w|^{2},
$$

for all $(x, \xi) \in \mathbb{R}^{2 n}$ with $\left|(x, \xi)-\left(x_{0}, \xi_{0}\right)\right| \leq \frac{1}{C_{2}}$ and all $w \in \mathbb{C}^{N}$. Here $\nabla_{x, \xi} H(x, \xi)=$ $\left(\partial_{x} H(x, \xi), \partial_{\xi} H(x, \xi)\right)$. If for some constants $C_{0}, C_{1}>0$ the above estimate holds for all $(x, \xi) \in \mathbb{R}^{2 n}$, we say that $H(x, \xi)$ is uniformly microhyperbolic on $\mathbb{R}^{2 n}$ in the direction $T$. In 
the case where $H(x, \xi)$ depends also on an additional parameter, we say that $H$ is uniformly microhyperbolic in the direction $T$ if (2.1) is satisfied with $C_{0}, C_{1}>0$ independent of this parameter.

\subsection{Trace formula for systems of $h$-pseudodifferential operators. Let}

$$
\theta \in C_{0}^{\infty}(]-1,1[; \mathbb{R}), \quad \theta_{\varepsilon}(t):=\theta(t / \varepsilon),
$$

where $\varepsilon>0$ is a positive constant possibly depending on $h$ and

$$
\mathcal{F}_{h}^{-1} \theta_{\varepsilon}(\tau)=\frac{1}{2 \pi h} \int_{\mathbb{R}} e^{i t \tau / h} \theta_{\varepsilon}(t) d t,
$$

the semiclassical Fourier inverse operator.

Let $A, H \in S^{0}\left(\mathbb{R}^{2 n} ; \mathcal{H}_{N}\right)$, and $\chi \in C_{0}^{\infty}\left(\mathbb{R}^{2 n} ; \mathbb{R}\right)$. We assume that $A^{w}\left(i+H^{w}\right)^{-k}$ is of trace class for some $k \in \mathbb{N}$. Writing $A^{w} f\left(H^{w}\right)=A^{w}\left(i+H^{w}\right)^{-k}\left(i+H^{w}\right)^{k} f\left(H^{w}\right)$ and using the fact that $\left(i+H^{w}\right)^{k} f\left(H^{w}\right)$ is bounded by the spectral theorem we deduce that $A^{w} f\left(H^{w}\right)$ is of trace class for all $f \in C_{0}^{\infty}(\mathbb{R} ; \mathbb{R})$. We recall that $\chi^{w}$ is of trace class (with norm trace $\mathcal{O}\left(h^{-n}\right)$, see [7. Theorem 9.4]).

Fix $\tau_{0} \in \mathbb{R}$. We denote by $O_{\tau_{0}}$ the set of open intervals centered at $\tau_{0}$, i.e.,

$$
O_{\tau_{0}}=\{] \tau_{0}-\eta, \tau_{0}+\eta[; \quad \eta>0\} .
$$

Theorem 2.2. Suppose that there exists $T \in \mathbb{R}^{2 n}$ such that $\tau_{0}-H(x, \xi)$ is uniformly microhyperbolic with respect to $(x, \xi) \in \mathbb{R}^{2 n}$ in the direction $T$. If $0 \notin \operatorname{supp} \theta$, then there exists $I \in O_{\tau_{0}}$ such that for all $f \in C_{0}^{\infty}(I ; \mathbb{R})$ and $\varepsilon \in\left[h^{1-\delta}, \kappa[\right.$ with $\kappa>0,0<\delta \leq 1$ independent of $h$, we have, uniformly for $\tau \in \mathbb{R}$,

$$
\operatorname{tr}\left(A^{w} f\left(H^{w}\right) \mathcal{F}_{h}^{-1} \theta_{\varepsilon}\left(\tau-H^{w}\right)\right)=\mathcal{O}\left(h^{\infty}\right) .
$$

Theorem 2.3. Let $H_{0}, H_{1} \in S^{0}\left(\mathbb{R}^{2 n}, \mathcal{H}_{N}\right)$ be such that $H_{0}=H_{1}$ in a neighborhood of $\operatorname{supp} \chi$. Then there exists $\varepsilon>0$ small and independent of $h$ such that we have, uniformly for $\tau \in \mathbb{R}$,

$$
\operatorname{tr}\left(\chi^{w}\left[f\left(H_{j}^{w}\right) \mathcal{F}_{h}^{-1} \theta_{\varepsilon}\left(\tau-H_{j}^{w}\right)\right]_{0}^{1}\right)=\mathcal{O}\left(h^{\infty}\right) .
$$

The following result is a simple consequence of the above theorems.

Theorem 2.4. Suppose that $\tau_{0}-H(x, \xi)$ is microhyperbolic at every point $(x, \xi)$ in $\operatorname{supp} \chi$. If $\theta$ equals 1 near $t=0$, then there exist $I \in O_{\tau_{0}}$ and $\varepsilon>0$ small and independent of $h$ such that for $f \in C_{0}^{\infty}(I ; \mathbb{R})$, the following full asymptotic expansion in powers of $h$ holds uniformly for $\tau \in \mathbb{R}$ :

$$
\operatorname{tr}\left(\chi^{w} f\left(H^{w}\right) \mathcal{F}_{h}^{-1} \theta_{\varepsilon}\left(\tau-H^{w}\right)\right) \sim(2 \pi h)^{-n} f(\tau) \sum_{j \geq 0} \gamma_{j}(\tau) h^{j} \quad \text { as } h \searrow 0 .
$$

Remark 2.2. The coefficients $\tau \mapsto \gamma_{j}(\tau)$ are smooth, independent of $f$ and $\theta$ and can be computed explicitly (see formula (3.25)).

2.2. Application to Schrödinger operators with matrix-valued potentials. In this section we apply the above trace formula to study the spectral properties of multi-channel semiclassical Schrödinger operators of the form

$$
P_{1}(h):=-h^{2} \Delta \otimes I_{N}+V(x), \quad P_{0}(h):=-h^{2} \Delta \otimes I_{N}+V_{\infty}, \quad \text { in } L^{2}\left(\mathbb{R}^{n} ; \mathbb{C}^{N}\right),
$$


where $I_{N}$ is the $N \times N$ identity matrix and $V(x)$ is a smooth hermitian matrix-valued potential, i.e.,

$$
V(x)=\left(V_{i j}(x)\right)_{1 \leq i, j \leq N}, V_{i j}(x)=\overline{V_{j i}(x)} .
$$

We assume that the matrix $V$ has a limit $V_{\infty}$ at infinity and

$$
\exists \mu>n \text { s.t. }\left\|\partial_{x}^{\alpha}\left(V(x)-V_{\infty}\right)\right\|_{N \times N}=\mathcal{O}_{\alpha}\left(\langle x\rangle^{-\mu-|\alpha|}\right), \quad \forall \alpha \in \mathbb{N}^{n}, \forall x \in \mathbb{R}^{n} .
$$

After a linear transformation, we may assume that

$$
V_{\infty}=\left(\begin{array}{cccc}
e_{1, \infty} & 0 & \cdots & 0 \\
0 & e_{2, \infty} & \cdots & 0 \\
\vdots & 0 & \ddots & \vdots \\
0 & \cdots & 0 & e_{N, \infty}
\end{array}\right) \text {, with } e_{1, \infty} \leq e_{2, \infty} \leq \cdots \leq e_{N, \infty}
$$

The operator $P_{0}(h)$ with domain $H^{2}\left(\mathbb{R}^{n} ; \mathbb{C}^{N}\right)$ is self-adjoint. Its spectrum is $\left[e_{1, \infty},+\infty[\right.$. Since $V-V_{\infty}$ is $\Delta$-compact, the operator $P_{1}(h)$ admits a unique self-adjoint realization in $L^{2}\left(\mathbb{R}^{n} ; \mathbb{C}^{N}\right)$ with domain $H^{2}\left(\mathbb{R}^{n} ; \mathbb{C}^{N}\right)$. Moreover the essential spectra of $P_{1}(h)$ and $P_{0}(h)$ are the same. The operator $P_{1}(h)$ may have discrete eigenvalues in $\left(-\infty, e_{1, \infty}\right)$ and embedded ones in the interval $\left[e_{1, \infty}, e_{N, \infty}\right]$ contained in the continuous spectrum.

The spectral shift function $s_{h}(\tau)$ associated to $\left(P_{1}(h), P_{0}(h)\right)$ is defined as a real-valued function on $\mathbb{R}$ satisfying the Lifshits-Krein formula

$$
\left\langle s_{h}^{\prime}(\cdot), f(\cdot)\right\rangle=-\operatorname{tr}\left(f\left(P_{1}(h)\right)-f\left(P_{0}(h)\right)\right), \quad \forall f \in C_{0}^{\infty}(\mathbb{R} ; \mathbb{R}) .
$$

The function $s_{h}(\tau)$ is fixed up to an additive constant by the formula (2.8), and we normalize it so that $s_{h}(\tau)=0$ for $\tau<\inf \left(\sigma\left(P_{1}(h)\right)\right.$.

We denote by $p_{1}(x, \xi):=\xi^{2} I_{N}+V(x)$ and $p_{0}(x, \xi):=\xi^{2} I_{N}+V_{\infty},(x, \xi) \in \mathbb{R}^{2 n}$, the classical Hamiltonians associated with the operators $P_{1}(h)$ and $P_{0}(h)$, respectively. Let $e_{1}(x) \leq e_{2}(x) \leq \ldots \leq e_{N}(x)$ be the eigenvalues of $V(x)$ arranged in increasing order.

Theorem 2.5. Assume (2.7) and let $f \in C_{0}^{\infty}(\mathbb{R} ; \mathbb{R})$. There exists a sequence of real numbers $\left(c_{2 j}(f)\right)_{j \in \mathbb{N}}$ such that

$$
\left\langle s_{h}^{\prime}(\cdot), f(\cdot)\right\rangle \sim(2 \pi h)^{-n} \sum_{j \geq 0} c_{2 j}(f) h^{2 j} \quad \text { as } h \searrow 0,
$$

with

$$
c_{0}(f)=\frac{\omega_{n}}{2} \sum_{k=1}^{N} \int_{\mathbb{R}^{n}} \int_{0}^{+\infty}\left[f\left(e_{k, \infty}+\tau\right)-f\left(e_{k}(x)+\tau\right)\right] \tau^{\frac{n-2}{2}} d \tau d x,
$$

where $\omega_{n}$ is the volume of the unit sphere $\mathbb{S}^{n-1}$.

For $\tau_{0} \in \mathbb{R}$, set

$$
\Sigma_{\tau_{0}}:=\bigcup_{k=1}^{N}\left\{(x, \xi) \in \mathbb{R}^{2 n} ; \xi^{2}+e_{k}(x)=\tau_{0}\right\} .
$$

The following theorem is a consequence of Theorem 2.4. 
Theorem 2.6 (Weak asymptotics). Let $\tau_{0} \notin\left\{e_{1, \infty}, e_{2, \infty}, \cdots, e_{N, \infty}\right\}$. Assume (2.7) and $\tau_{0}-p_{1}(x, \xi)$ is microhyperbolic at every point $(x, \xi) \in \Sigma_{\tau_{0}}$. Then, if $\theta$ is equal to 1 near the origin, there exist $I \in O_{\tau_{0}}$ and $\varepsilon$ small enough and independent of $h$ such that for $f \in$ $C_{0}^{\infty}(I ; \mathbb{R})$, the following asymptotic formula holds uniformly for $\tau \in \mathbb{R}$ :

$$
\left\langle s_{h}^{\prime}(\cdot), \mathcal{F}_{h}^{-1} \theta_{\varepsilon}(\tau-\cdot) f(\cdot)\right\rangle \sim(2 \pi h)^{-n} f(\tau) \sum_{j \geq 0} \gamma_{2 j}(\tau) h^{2 j} \quad \text { as } h \searrow 0 .
$$

The coefficients $\gamma_{2 j}(\tau)$ are smooth functions of $\tau$, independent of $f$ and $\theta$. In particular,

$$
\gamma_{0}(\tau)=\frac{\omega_{n}}{2} \sum_{k=1}^{N} \int_{\mathbb{R}^{n}}\left(\left(\tau-e_{k}(x)\right)_{+}^{\frac{n-2}{2}}-\left(\tau-e_{k, \infty}\right)_{+}^{\frac{n-2}{2}}\right) d x,
$$

where $\tau_{+}:=\max (\tau, 0)$.

Remark 2.3. According to Definition 2.1, the assumption that $\tau_{0}-p_{1}(x, \xi)$ is microhyperbolic at every point $(x, \xi) \in \Sigma_{\tau_{0}}$ is equivalent to the following condition: For $x_{0}$ with $e_{j}\left(x_{0}\right)=\tau_{0}, j=1, \ldots, N$, there exists $T_{1} \in \mathbb{R}^{n}$ and $C>0$ such that

$$
\left(\left\langle T_{1}, \nabla_{x} V\left(x_{0}\right)\right\rangle \omega, \omega\right) \geq \frac{1}{C}|\omega|^{2}, \forall \omega \in \operatorname{ker}\left(V\left(x_{0}\right)-\tau_{0} I_{N}\right) .
$$

In particular, if $e_{j}\left(x_{0}\right)$ is a simple eigenvalue of $V\left(x_{0}\right)$, this is equivalent to $\nabla e_{j}\left(x_{0}\right) \neq 0$.

As a consequence of Theorem 2.6. we get a sharp remainder estimate for the spectral shift function corresponding to the pair $\left(P_{1}(h), P_{0}(h)\right)$.

Theorem 2.7 (Weyl-type asymptotics). Assume that (2.7) holds with $V_{\infty}=0$. Let $\tau_{0} \neq 0$ such that $\tau_{0}-p_{1}(x, \xi)$ is microhyperbolic at every point $(x, \xi) \in \Sigma_{\tau_{0}}$. There exists $I \in O_{\tau_{0}}$ such that

$$
s_{h}(\tau)=(2 \pi h)^{-n} a_{0}(\tau)+\mathcal{O}\left(h^{-n+1}\right) \quad \text { as } h \searrow 0,
$$

uniformly for $\tau \in I$, with

$$
a_{0}(\tau)=\frac{\omega_{n}}{n} \sum_{k=1}^{N} \int_{\mathbb{R}^{n}}\left(\left(\tau-e_{k}(x)\right)_{+}^{\frac{n}{2}}-\tau_{+}^{\frac{n}{2}}\right) d x .
$$

As indicated in the introduction, in the scalar case a complete asymptotic expansion in powers of $h$ of the derivative of the SSF has been obtained under a non-trapping condition on the classical trajectories corresponding to the energy surface $\Sigma_{\tau_{0}}$ (see [30]). In the present matrix-valued case, the treatment is much more complicated. In fact, since the eigenvalues are not enough regular, the usual definition of the Hamilton flow for a matrix-valued Hamiltonian function does not make sense (see [16]). For this reason, we use here the notion of escape function.

More precisely, we suppose that there exists a scalar escape function $G \in C^{\infty}\left(\mathbb{R}^{2 n} ; \mathbb{R}\right)$ associated to $p_{1}$ at $\tau_{0}$, i.e.,

$$
\exists C>0, \text { s.t. }\left\{p_{1}, G\right\}(x, \xi):=\frac{\partial G}{\partial x} \cdot \frac{\partial p_{1}}{\partial \xi}-\frac{\partial G}{\partial \xi} \cdot \frac{\partial p_{1}}{\partial x} \geq C, \quad \forall(x, \xi) \in \Sigma_{\tau_{0}},
$$

in the sense of hermitian matrices.

In the scalar case $N=1$, it is well known that the above assumption is equivalent to the non-trapping condition on the energy $\tau_{0}$. In fact, if $\tau_{0}$ is non-trapping for the classical 
Hamiltonian $p_{1}$, one can construct an escape function $G \in C^{\infty}\left(\mathbb{R}^{2 n} ; \mathbb{R}\right)$ satisfying (2.15) (see for instance [9], 33], 34], 35]). Conversely, if (2.15) holds then one easily sees that $G$ is strictly increasing along the Hamiltonian flows associated to $p_{1}$ in $\Sigma_{\tau_{0}}$ which prevents the existence of trapped trajectories at $\tau_{0}$. We also point out that (2.15) implies that $\tau_{0}-p_{1}(x, \xi)$ is microhyperbolic at every point $(x, \xi) \in \Sigma_{\tau_{0}}$ in the direction of the Hamiltonian vector field $\left(\partial_{\xi} G(x, \xi),-\partial_{x} G(x, \xi)\right)$.

Now we can formulate the main result of this paper.

Theorem 2.8 (Strong asymptotics). Fix an energy $\tau_{0}>e_{N, \infty}$. Assume that (2.7) and (2.15) are satisfied. Then, there exists $I \in O_{\tau_{0}}$ such that $s_{h}^{\prime}(\cdot)$ has a complete asymptotic expansion of the form

$$
s_{h}^{\prime}(\tau) \sim(2 \pi h)^{-n} \sum_{j \geq 0} \gamma_{2 j}(\tau) h^{2 j} \quad \text { as } h \searrow 0,
$$

uniformly for $\tau \in I$, where the coefficients $\gamma_{2 j}(\tau)$ are given in Theorem 2.6.

2.3. Examples and further generalizations. First observe that, for $G(x, \xi)=x \cdot \xi$, (2.15) is equivalent to

$$
2\left(\tau_{0}-e_{k}(x)\right)-x \cdot \nabla V(x) \geq C, \quad \forall x \in\left\{x \in \mathbb{R}^{n} ; \tau_{0}-e_{k}(x) \geq 0\right\}, k=1, \cdots N .
$$

Thus, under the assumption (2.7), the asymptotics (2.16) holds near any large $\tau_{0}$ with

$$
\tau_{0}>\sup _{x \in \mathbb{R}^{n}}\left\|\frac{x \cdot \nabla V(x)}{2}\right\|_{N \times N}+\sup _{x \in \mathbb{R}^{n}}\|V(x)\|_{N \times N} .
$$

Notice that our results extend to the case of potentials depending on $h$, i.e. $V(x ; h)=$ $V_{0}(x)+h V_{1}(x ; h)$. In such a case, we assume (2.7) uniformly with respect to $h$. In particular, as a simple example, consider the case where $V_{0}(x)$ is a diagonal matrix diag $\left(e_{1}(x), \ldots, e_{N}(x)\right)$. If each $e_{j}(x)$ satisfies

$$
2\left(\tau_{0}-e_{j}(x)\right)-x \cdot \nabla_{x} e_{j}(x) \geq c_{j}>0, \quad \forall x \in\left\{x \in \mathbb{R}^{n} ; \tau_{0}-e_{j}(x) \geq 0\right\},
$$

then (2.17) is satisfied for $h$ small enough and (2.16) holds.

More generally, we can treat the spectral shift function associated to a pair of self-adjoint $h$-pseudodifferential operators $\left(P_{1}(h), P_{0}(h)\right)$ provided that the SSF is well defined and the existence of a scalar escape function holds.

2.4. Outline of the proofs. The purpose of this subsection is to provide an outline of the proofs.

As indicated in the introduction, our method is time-independent. The starting point is the functional calculus of $h$-pseudodifferential operators based on the Helffer-Sjöstrand formula (see [7, Ch. 8]). By this formula, the main object to study will be the integral of the form

$$
\mathcal{I}(\tau, \varepsilon ; h)=-\frac{1}{\pi} \int_{\mathbb{C}} \bar{\partial} \tilde{f}(z) \mathcal{F}_{h}^{-1} \theta_{\varepsilon}(\tau-z) K(z ; h) L(d z), \quad \tau \in \mathbb{R}
$$

where $L(d z)=d x d y$ is the Lebesgue measure on $\mathbb{C} \sim \mathbb{R}^{2}$. Here, $\tilde{f} \in C_{0}^{\infty}(\mathbb{C})$ denotes an almost analytic extension of $f \in C_{0}^{\infty}(\mathbb{R} ; \mathbb{R})$ (see [7, Ch. 8] and also [11]), i.e.,

$$
\begin{gathered}
\tilde{f}_{\mid \mathbb{R}}=f, \\
\bar{\partial} \tilde{f}(z)=\mathcal{O}\left(|\Im z|^{\infty}\right),
\end{gathered}
$$


and $K$, which in fact is the trace of an operator depending on the resolvent, is a complexvalued analytic function defined in a neighborhood of supp $\tilde{f}$ except on the real axis, with an estimate

$$
K(z ; h)=\mathcal{O}\left(h^{-n}|\Im z|^{-2}\right) .
$$

The right hand side of (2.18) is independent of the particular choice of the almost analytic extension $\tilde{f}$. In particular, let $\psi_{L}(z)$ be a function on $\mathbb{C}$ defined by

$$
\psi_{L}(z)=\psi\left(\frac{\Im z}{L}\right), \quad L>0, \quad C_{0}^{\infty}(\mathbb{R} ; \mathbb{R}) \ni \psi(t)=\left\{\begin{array}{cc}
1 & (|t| \leq 1) \\
0 & (|t| \geq 2) .
\end{array}\right.
$$

Then $\tilde{f} \psi_{L}$ is also an almost analytic extension of $f$, and we have

$$
\mathcal{I}(\tau, \varepsilon ; h)=-\frac{1}{\pi} \int_{\mathbb{C}} \bar{\partial}\left(\tilde{f} \psi_{L}\right)(z) \mathcal{F}_{h}^{-1} \theta_{\varepsilon}(\tau-z) K(z ; h) L(d z) .
$$

From now on, $M>0$ is a constant independent of $h$ and we put

$$
\zeta(h):=h \log \left(\frac{1}{h}\right), \quad L:=\frac{M \zeta(h)}{\varepsilon} .
$$

We begin with a general remark on the integral given by the right hand side of (2.23). From (2.20) and the definition of $\psi_{L}$, we deduce

$$
\bar{\partial}\left(\tilde{f} \psi_{L}\right)(z)=\mathcal{O}\left(h^{\infty}\right) \psi_{L}(z)+\mathcal{O}\left(\frac{1}{L}\right) \tilde{f}(z) 1_{[1,2] \cup[-2,-1]}\left(\frac{\Im z}{L}\right),
$$

which together with (2.27) yields $\mathcal{I}(\tau, \varepsilon ; h) \equiv \mathcal{I}_{+}(\tau, \varepsilon ; h)+\mathcal{I}_{-}(\tau, \varepsilon ; h)$, uniformly for $0<\varepsilon \leq$ $c h^{-\nu}$ (where $\nu$ is a fixed constant). Here

$$
\mathcal{I}_{ \pm}(\tau, \varepsilon ; h):=-\frac{1}{\pi} \int_{\{ \pm \Im z>L\}} \bar{\partial}\left(\tilde{f} \psi_{L}\right)(z) \mathcal{F}_{h}^{-1} \theta_{\varepsilon}(\tau-z) K(z ; h) L(d z) .
$$

We recall that the notation $A \equiv B$ means $A-B=\mathcal{O}\left(h^{\infty}\right)$. The behavior of the function $\mathcal{F}_{h}^{-1} \theta_{\varepsilon}(\tau-z)$ depends on the support of $\theta$. For general $\theta$ with support in $]-1,1[$, we have

$$
\mathcal{F}_{h}^{-1} \theta_{\varepsilon}(\tau-z)=\mathcal{O}\left(\frac{\varepsilon}{h} e^{\frac{\varepsilon|\Im z|}{h}}\right) .
$$

In particular, in the support of $\psi_{L}$, we have

$$
\mathcal{F}_{h}^{-1} \theta_{\varepsilon}(\tau-z)=\mathcal{O}\left(\varepsilon h^{-2 M-1}\right) .
$$

For $\theta$ with support only in $\mathbb{R}_{+}$, say in $] \frac{1}{2}, 1[$, we have

$$
\mathcal{F}_{h}^{-1} \theta_{\varepsilon}(\tau-z)= \begin{cases}\mathcal{O}\left(\frac{\varepsilon}{h} e^{\frac{\varepsilon \Im z}{h}}\right), & \Im z>0, \\ \mathcal{O}\left(\frac{\varepsilon}{h} e^{\frac{\varepsilon \Im z}{2 h}}\right), & \Im z<0 .\end{cases}
$$

This latter estimate implies in particular that

$$
\mathcal{I}_{-}(\tau, \varepsilon ; h)=\mathcal{O}\left(\varepsilon^{2} h^{\frac{M}{2}-n-2}\right),
$$

which means $\mathcal{I}_{-}=\mathcal{O}\left(h^{\infty}\right)$ if $\varepsilon$ is at most of polynomial order in $h$ and $M$ is arbitrary.

Let $\theta, \varepsilon$ be as in Theorem 2.2 and assume that $\tau_{0}-H(x, \xi)$ is uniformly microhyperbolic on $\mathbb{R}^{2 n}$ in the direction $T$. Without any loss of generality we may assume that $\theta \in C_{0}^{\infty}(] \frac{1}{2}, 1[; \mathbb{R})$. 
According to the Helffer-Sjöstrand formula (see (3.3), (3.4)), the left hand side of (2.3) can be written as (2.18) with

$$
K(z ; h):=\left(\lambda_{0}-z\right)^{k-1} \operatorname{tr}\left(A^{w}\left(z-H^{w}\right)^{-1}\left(\lambda_{0}-H^{w}\right)^{-(k-1)}\right),
$$

where $\lambda_{0}<\inf \left(\sigma\left(H^{w}\right)\right)$ and $k \in \mathbb{N}$ is large enough so that $A^{w}\left(z-H^{w}\right)^{-1}\left(\lambda_{0}-H^{w}\right)^{-(k-1)}$ is of trace class.

As explained above, we have $\mathcal{I}_{-}=\mathcal{O}\left(h^{\infty}\right)$. To deal with $\mathcal{I}_{+}$, we conjugate the operator $A^{w}\left(z-H^{w}\right)^{-1}\left(\lambda_{0}-H^{w}\right)^{-(k-1)}$ with the unitary operator $U_{t}:=e^{\frac{i t}{h}\left(T_{2} \cdot x-T_{1} \cdot h D_{x}\right)}, t \in \mathbb{R}$. Here $T=\left(T_{1}, T_{2}\right)$ is the direction of the uniform microhyperbolicity of $\tau_{0}-H(x, \xi)$. Then the function

$$
K_{t}(z ; h):=\left(\lambda_{0}-z\right)^{k-1} \operatorname{tr}\left(A_{t}^{w}\left(z-H_{t}^{w}\right)^{-1}\left(\lambda_{0}-H_{t}^{w}\right)^{-(k-1)}\right),
$$

with $H_{t}^{w}:=U_{t} H^{w}\left(x, h D_{x}\right) U_{t}^{-1}=H^{w}\left(x+t T_{1}, h D_{x}+t T_{2}\right)$ etc., is invariant with respect to the change of real $t$ and coincides with $K(z ; h)$ thanks to the cyclicity of the trace.

Now, replacing $H, A$ by their almost analytic extensions $(\tilde{H}$ and $\tilde{A})$, we extend this function to complex $t$. The extended function $\tilde{K}_{t}(z ; h)$ is defined in $\left\{z \in \mathbb{C} ; \Im z>C_{0} \Im t\right\}$ for some positive constant $C_{0}$ independent of $M, \varepsilon$ and $h$. We fix $t_{0}=i L / C_{0}$. Then we see that $\tilde{K}_{t_{0}}(z ; h)$ is equal to $K(z ; h)$ modulo $\mathcal{O}\left(h^{\infty}\right)$ in the domain $\{\Im z>L\}$.

The uniform microhyperbolic condition enables us to continue $\tilde{K}_{t_{0}}(z ; h)$ analytically to the lower half plane with $\Im z>-c L$ for a positive contant $c$. In fact, the imaginary part of the Weyl symbol of $z-\tilde{H}_{t_{0}}$ stays positive definite in such a region, and the sharp Gårding inequality guarantees the invertibility of the operator.

In the integral expression (2.26) of $\mathcal{I}_{+}$, we can replace, modulo $\mathcal{O}\left(h^{\infty}\right), K(z ; h)$ by $\tilde{K}_{t_{0}}(z ; h)$ and then the integral domain $\Im z>L$ by $\Im z<-c L$ by the Cauchy theorem. Thus the estimate $\mathcal{I}_{+}=\mathcal{O}\left(h^{\infty}\right)$ is reduced to the same argument as for $\mathcal{I}_{-}$, and we conclude $\mathcal{I}=\mathcal{O}\left(h^{\infty}\right)$. This gives Theorem 2.2

Let us now outline the proof of Theorem 2.3. By Helffer-Sjöstrand formula, the left hand side of (2.4) can be written as (2.18) with $K(z ; h)=\operatorname{tr}\left(\chi^{w}\left[\left(z-H_{j}^{w}\right)^{-1}\right]_{0}^{1}\right)$. Using that $\operatorname{dist}\left(\operatorname{supp} \chi, \operatorname{supp}\left(H_{0}-H_{1}\right)\right)>0$, we prove by some exponentially weighted resolvent estimates

$$
K(z ; h)=\mathcal{O}\left(\frac{h^{\frac{M}{\varepsilon C}}-n}{L^{2}}\right),
$$

uniformly for $|\Im z| \geq L$, where $C>0$ is a constant independent of $h, M, \varepsilon$. Combining this with (2.28), we get Theorem 2.3 provided that $\varepsilon>0$ is small enough.

Theorem 2.4 is a consequence of the two previous theorems and the symbolic calculus of $h$-pseudodifferential operators. Assuming that $\chi$ is supported in a small neighborhood of a fixed point $\left(x_{0}, \xi_{0}\right) \in \mathbb{R}^{2 n}$ (by a partition of unity there is no loss of generality in doing so) and using the fact that changing $H$ outside the support of $\chi$ leads to an error of order $\mathcal{O}\left(h^{\infty}\right)$ in the trace formula $\operatorname{tr}\left(\chi^{w} f\left(H^{w}\right) \mathcal{F}_{h}^{-1} \theta\left(\tau-H^{w}\right)\right.$ ) (according to Theorem 2.3), together with Theorem A.3, we may assume that there exists $I \in O_{\tau_{0}}$ such that $\tau-H$ is uniformly microhyperbolic with respect to $(x, \xi) \in \mathbb{R}^{2 n}$ and $\tau \in I$.

Now, fix $\tilde{\varepsilon}=h^{1-\delta_{0}}$ with $\left.\delta_{0} \in\right] 0,1 / 2[$. Applying Theorem 2.2, we obtain

$$
\operatorname{tr}\left(\chi^{w} f\left(H^{w}\right) \mathcal{F}_{h}^{-1} \theta\left(\tau-H^{w}\right)\right) \equiv \operatorname{tr}\left(\chi^{w} f\left(H^{w}\right) \mathcal{F}_{h}^{-1} \theta_{\tilde{\varepsilon}}\left(\tau-H^{w}\right)\right) .
$$


In fact we can represent the difference $\theta-\theta_{\tilde{\varepsilon}}$ as a finite sum of functions $\tilde{\theta}_{\varepsilon}$ appearing in Theorem 2.2 (with $\varepsilon \in\left[\tilde{\varepsilon}, \frac{1}{C}[\right.$ ). The principal significance of (2.31) is that it allows one to use the standard $h$-pseudodifferential calculus and get the asymptotic expansion in powers of $h$ given in Theorem 2.4 just by symbolic calculus (see [7, Ch. 7-8]) . To see this, we first recall that for $|\Im z|>h^{\delta}$ (with $\left.\delta \in\right] 0,1 / 2\left[\right.$ ) the resolvent $\left(z-H^{w}\right)^{-1}$ is an $h$-pseudodifferential operator and its corresponding symbol admits an asymptotic expansion in powers of $h$ (see (3.21) ). Combining this with the fact

$$
\begin{gathered}
\operatorname{tr}\left(\chi^{w} f\left(H^{w}\right) \mathcal{F}_{h}^{-1} \theta_{\tilde{\varepsilon}}\left(\tau-H^{w}\right)\right) \\
\equiv-\frac{1}{\pi} \int_{\left\{|\Im z|>h^{\delta_{0}}\right\}} \bar{\partial}\left(\tilde{f} \psi\left(\frac{\Im z}{h^{\delta_{0}}}\right)\right)(z) \mathcal{F}_{h}^{-1} \theta_{\tilde{\varepsilon}}(\tau-z) \operatorname{tr}\left(\chi^{w}\left(z-H^{w}\right)^{-1}\right) L(d z),
\end{gathered}
$$

we see that the left hand side of (2.31) has a complete asymptotic expansion in powers of $h$, which yields Theorem 2.4.

Turn now to the main ideas in the proofs of the results of subsection 2.2 concerning our application to the SSF. Theorem 2.5 is a simple consequence of the $h$-pseudodifferential symbolic calculus while Theorems 2.6 and 2.7 are consequences of Theorem 2.4 and standard Tauberian arguments combined with a trick of Robert [27] respectively.

Finally, we sketch the proof of our main result which is Theorem 2.8, According to (2.8) and the Helffer-Sjöstrand formula we have

$$
-\left\langle s_{h}^{\prime}(\cdot), \mathcal{F}_{h}^{-1} \theta_{\varepsilon}(\tau-\cdot) f(\cdot)\right\rangle=\mathcal{I}(\tau, \varepsilon ; h),
$$

with

$$
K(z ; h)=\left(z-\lambda_{0}\right)^{q} \operatorname{tr}\left(\left[\left(P_{j}(h)-\lambda_{0}\right)^{-q}\left(P_{j}(h)-z\right)^{-1}\right]_{0}^{1}\right) .
$$

Here $\lambda_{0}<\inf \sigma\left(P_{1}(h)\right)$ and $q \in \mathbb{N}$ is large enough, see (4.3).

First, suppose that 0 is not contained in the support of $\theta$. Then, $\mathcal{I}_{-}(\tau, \varepsilon ; h)=\mathcal{O}\left(h^{\infty}\right)$ uniformly for $\varepsilon \in] h^{1-\delta}, h^{-\nu}[$ as before.

To deal with $\mathcal{I}_{+}(\tau, \varepsilon ; h)$, we adapt an idea from the theory of resonance. More precisely, under the existence of an escape function near $\tau_{0}$ (assumption (2.15)), we prove by the analytic distortion method that $K(z ; h)$ extends analytically from the upper half plane to the lower one with $\Im z>-M \zeta(h)$ for all $M>0$.

From this, we deduce two important consequences. First,

$$
s_{h}^{\prime \prime}(\tau)=\mathcal{O}\left(h^{-n} \zeta(h)^{-2}\right) .
$$

uniformly for $\tau$ near $\tau_{0}$. Second, the same argument as in the proof of Theorem 2.2 leads to $\mathcal{I}_{+}(\tau, \varepsilon ; h)=\mathcal{O}\left(h^{\infty}\right)$, uniformly for $\left.\varepsilon \in\right] h^{1-\delta}, h^{-\nu}[$. Hence we obtain

$$
\left\langle s_{h}^{\prime}(\cdot), \mathcal{F}_{h}^{-1} \theta_{\varepsilon}(\tau-\cdot) f(\cdot)\right\rangle=\mathcal{O}\left(h^{\infty}\right) .
$$

Now, we assume that $\theta$ is equal to one near zero, and let $\varepsilon$ be small and independent of $h$ and $\tilde{\varepsilon}=h^{-\nu}$. As in the proof of (2.31), the formula (2.33) yields

$$
\left\langle s_{h}^{\prime}(\cdot), \mathcal{F}_{h}^{-1} \theta_{\varepsilon}(\tau-\cdot) f(\cdot)\right\rangle \equiv\left\langle s_{h}^{\prime}(\cdot), \mathcal{F}_{h}^{-1} \theta_{\tilde{\varepsilon}}(\tau-\cdot) f(\cdot)\right\rangle .
$$

By (2.8) and (2.11), the left hand side of the above equality has an asymptotic expansion in powers of $h$. The right hand side is written, by Taylor's formula and (2.32),

$$
\left\langle s_{h}^{\prime}(\cdot), \mathcal{F}_{h}^{-1} \theta_{\varepsilon}(\tau-\cdot) f(\cdot)\right\rangle=s_{h}^{\prime}(\tau) f(\tau)+\mathcal{O}\left(h^{\nu+1-n} \zeta(h)^{-2}\right) .
$$


Since $\nu$ is arbitrary, this ends the proof of Theorem 2.8 by taking $f=1$ near $\tau_{0}$.

\section{Proofs of the Results on the Semiclassical trace Formula}

In this section, we prove the results concerning the semiclassical trace formula. Throughout our proofs, when it is not precised, we let $C$ denotes a positive constant that may take different values, but is always independent of $\varepsilon, h$ and $M$.

3.1. Proof of Theorem 2.2. Writing $\theta=\theta_{1}+\theta_{2}$, with supp $\left.\theta_{1} \subset\right] 0,+\infty\left[\right.$ and $\operatorname{supp} \theta_{2} \subset$ ]$-\infty, 0[$, we may assume that $\operatorname{supp} \theta \subset] \frac{1}{2}, 1[$.

For $\tau \in \mathbb{R}$ and $\varepsilon>0$, we define

$$
\mathcal{I}(\tau, \varepsilon ; h):=\operatorname{tr}\left(A^{w} f\left(H^{w}\right) \mathcal{F}_{h}^{-1} \theta_{\varepsilon}\left(\tau-H^{w}\right)\right) .
$$

Let $\tilde{f}$ be an almost analytic extension of $f$ satisfying (2.19) and (2.20) with $\operatorname{supp} \tilde{f} \subset\{z \in$ $\mathbb{C} ;|\Im z| \leq 1\}$. If $g$ is real analytic in a neighborhood of the support of $\tilde{f}$, then we have, by the Helffer-Sjöstrand formula (see [7, Ch. 8]),

$$
f\left(H^{w}\right) g\left(H^{w}\right)=-\frac{1}{\pi} \int_{\mathbb{C}} \bar{\partial} \tilde{f}(z) g(z)\left(z-H^{w}\right)^{-1} L(d z) .
$$

Let $\lambda_{0} \in \mathbb{R}$ be fixed such that $\lambda_{0}<\inf \left(\sigma\left(H^{w}\right)\right)$ and set, for $\Im z \neq 0$,

$$
K(z ; h):=\left(\lambda_{0}-z\right)^{k-1} \operatorname{tr}\left(A^{w}\left(z-H^{w}\right)^{-1}\left(\lambda_{0}-H^{w}\right)^{-(k-1)}\right) .
$$

Then, using (3.1), (3.2) and (3.3) with $g(z)=\left(\lambda_{0}-z\right)^{k-1} \mathcal{F}_{h}^{-1} \theta_{\varepsilon}(\tau-z)$, we obtain

$$
\mathcal{I}(\tau, \varepsilon ; h)=-\frac{1}{\pi} \int_{\mathbb{C}} \bar{\partial} \tilde{f}(z) \mathcal{F}_{h}^{-1} \theta_{\varepsilon}(\tau-z) K(z ; h) L(d z) .
$$

Let $L$ and $\psi_{L}$ be defined by (2.24) and (2.22). We write

$$
\mathcal{I}=\mathcal{I}_{+}+\mathcal{I}_{-}, \quad \mathcal{I}_{ \pm}:=-\frac{1}{\pi} \int_{\{ \pm \Im z>0\}} \bar{\partial}\left(\tilde{f} \psi_{L}\right)(z) \mathcal{F}_{h}^{-1} \theta_{\varepsilon}(\tau-z) K(z ; h) L(d z) .
$$

Since the support of $\theta$ is included in $] \frac{1}{2}, 1[$, it follows from (2.30) that

$$
\mathcal{I}_{-}(\tau, \varepsilon ; h) \equiv 0,
$$

uniformly for $\tau \in \mathbb{R}$ and $\varepsilon \in\left[h^{1-\delta}, \kappa[\right.$, for all $\kappa>0$.

Let us now turn to the study of $\mathcal{I}_{+}(\tau, \varepsilon ; h)$. By assumption, there exists $T=\left(T_{1}, T_{2}\right) \in \mathbb{R}^{2 n}$ and $I_{\tau_{0}} \in O_{\tau_{0}}$ such that $\tau-H(x, \xi)$ is uniformly microhyperbolic in the direction $T$ with respect to $(x, \xi) \in \mathbb{R}^{2 n}$ and $\tau \in I_{\tau_{0}}$.

For $t \in \mathbb{R}$, we define the unitary operator

$$
U_{t}:=e^{\frac{i t}{h}\left(T_{2} \cdot x-T_{1} \cdot h D_{x}\right)} .
$$

Clearly, we have

$$
\begin{aligned}
H_{t}^{w} & :=U_{t}^{-1} H^{w}\left(x, h D_{x}\right) U_{t}=H^{w}\left(\left(x, h D_{x}\right)+t T\right)=H^{w}\left(x+t T_{1}, h D_{x}+t T_{2}\right), \\
A_{t}^{w} & :=U_{t}^{-1} A^{w}\left(x, h D_{x}\right) U_{t}=A^{w}\left(\left(x, h D_{x}\right)+t T\right)=A^{w}\left(x+t T_{1}, h D_{x}+t T_{2}\right) .
\end{aligned}
$$

Let $\tilde{H}, \tilde{A}$ be two almost analytic extensions of $H$ and $A$, respectively, which are bounded together with all theirs derivatives. Put for complex $t$ with small imaginary part

$$
\tilde{H}_{t}^{w}:=\tilde{H}^{w}\left(\left(x, h D_{x}\right)+t T\right) \quad \text { and } \quad \tilde{A}_{t}^{w}:=\tilde{A}^{w}\left(\left(x, h D_{x}\right)+t T\right) .
$$


By Taylor's formula with respect to $\Im t$, we have

$z-\tilde{H}((x, \xi)+t T)=z-\tilde{H}((x, \xi)+\Re t T+i \Im t T)$

$$
=z-H((x, \xi)+\Re t T)-i \Im t\left\langle\nabla_{x, \xi} H((x, \xi)+\Re t T), T\right\rangle+\mathcal{O}\left(|\Im t|^{2}\right) .
$$

Thus, one easily sees by using the Calderón-Vaillancourt theorem (see [7, Theorem 7.11]) that there exists a constant $C_{0}>0$ (depending only on the $L^{\infty}$-norms of a finite numbers of derivatives of $H)$ such that $\left(z-\tilde{H}_{t}^{w}\right)^{-1}$ exists for $|\Im z| \geq C_{0}|\Im t|$. Set

$$
\tilde{K}_{t}(z ; h):=\left(\lambda_{0}-z\right)^{k-1} \operatorname{tr}\left(\tilde{A}_{t}^{w}\left(z-\tilde{H}_{t}^{w}\right)^{-1}\left(\lambda_{0}-\tilde{H}_{t}^{w}\right)^{-(k-1)}\right) .
$$

Using that $\overline{\partial_{t}} \tilde{A}_{t}, \bar{\partial}_{t} \tilde{H}_{t}=\mathcal{O}\left(|\Im t|^{\infty}\right)$, we obtain, uniformly on $\left\{z \in \mathbb{C} ;|\Im z| \geq C_{0}|\Im t|\right\}$,

$$
\bar{\partial}_{t} \tilde{K}_{t}(z ; h)=\mathcal{O}\left(\frac{|\Im t|^{\infty}}{|\Im z|^{2}}\right)
$$

On the other hand, since $U_{t}$ is unitary for $t \in \mathbb{R}$, it follows from the cyclicity of the trace that $\tilde{K}_{t}$ is independent of $\Re t$. This implies

$$
\bar{\partial}_{t} \tilde{K}_{t}(z ; h)=\frac{i}{2} \partial_{\Im t} \tilde{K}_{t}(z ; h), \quad \text { and } \quad \tilde{K}_{t}(z ; h)=K(z ; h), \quad \forall t \in \mathbb{R} .
$$

We have, uniformly for $|\Im z| \geq C_{0}|\Im t|$,

$$
K(z ; h)-\tilde{K}_{i \Im t}(z ; h)=\tilde{K}_{\Re t}(z ; h)-\tilde{K}_{\Re t+i \Im t}(z ; h)=-\int_{0}^{\Im t} \frac{d}{d s} \tilde{K}_{\Re t+i s}(z ; h) d s=\mathcal{O}\left(\frac{|\Im t|^{\infty}}{|\Im z|^{2}}\right) .
$$

Fix $t_{0}=i L / C_{0}$. By the preceding estimate, we have, uniformly for $|\Im z| \geq L$,

$$
K(z ; h)-\tilde{K}_{t_{0}}(z ; h)=\mathcal{O}\left(h^{\infty}\right) .
$$

In the expression (3.5) of $I_{+}$, one sees from (2.25) and (2.29) that the restriction of the integral to the domain $0<\Im z \leq L$ is $\mathcal{O}\left(h^{\infty}\right)$. Therefore, by (3.10), we get

$$
\begin{aligned}
\mathcal{I}_{+}(\tau, \varepsilon ; h) & \equiv-\frac{1}{\pi} \int_{\{\Im z>L\}} \bar{\partial}\left(\tilde{f} \psi_{L}\right)(z) \mathcal{F}_{h}^{-1} \theta_{\varepsilon}(\tau-z) K(z ; h) L(d z) \\
& \equiv-\frac{1}{\pi} \int_{\{\Im z>L\}} \bar{\partial}\left(\tilde{f} \psi_{L}\right)(z) \mathcal{F}_{h}^{-1} \theta_{\varepsilon}(\tau-z) \tilde{K}_{t_{0}}(z ; h) L(d z) .
\end{aligned}
$$

Lemma 3.1. Let $t_{0}=i L / C_{0}=\frac{i M}{C_{0} \varepsilon} \zeta(h)$ be as above. The function $z \mapsto \tilde{K}_{t_{0}}(z ; h)$ extends as a holomorphic function to the zone $\Im z \geq-\frac{\left|t_{0}\right|}{2}$.

Proof. As in (3.7), Taylor's formula yields

$$
z-\tilde{H}_{t_{0}}(x, \xi)=z-H(x, \xi)-t_{0}\left\langle T, \nabla_{x, \xi} H(x, \xi)\right\rangle+\mathcal{O}\left(\left|t_{0}\right|^{2}\right) .
$$

Using the global microhyperbolicity condition, we obtain for small $h$

$$
\Im\left(z-\tilde{H}_{t_{0}}(x, \xi)\right)+C\left|t_{0}\right|\left(z-\tilde{H}_{t_{0}}(x, \xi)\right)^{*}\left(z-\tilde{H}_{t_{0}}(x, \xi)\right) \geq c\left(\left|t_{0}\right|+\Im z\right) I_{N},
$$

uniformly on $z$ with $\Im z>0$ and $\Re z \in I$ (see (A.8)), where $C, c>0$ are constants independent of $h$ and $M$. Here, * stands for the usual complex adjoint of a matrix.

Now we pass from the symbolic calculus level to the $h$-pseudodifferential calculus. The semiclassical version of the sharp Gårding inequality (see [7] Theorem 7.12 and [14, Ch.1] for the matrix case) and (3.11) imply,

$$
\Im\left(\mathrm{Op}_{h}^{w}\left(z-\tilde{H}_{t_{0}}\right) u, u\right)+C\left|t_{0}\right|\left\|\mathrm{Op}_{h}^{w}\left(z-\tilde{H}_{t_{0}}\right) u\right\|^{2}
$$




$$
\geq c\left(\left|t_{0}\right|+\Im z\right)\|u\|^{2}-\mathcal{O}(h)\|u\|^{2} \geq \frac{c}{3}\left(\left|t_{0}\right|+\Im z\right)\|u\|^{2},
$$

for all $u \in L^{2}\left(\mathbb{R}^{n} ; \mathbb{C}^{N}\right)$ and $h$ small enough. Here we used the fact that $h=o\left(\left|t_{0}\right|\right)$. Combining (3.12) with the inequality $a b \leq \frac{c\left|t_{0}\right|}{6} a^{2}+\frac{3}{2 c\left|t_{0}\right|} b^{2}$, we obtain

$$
\begin{gathered}
\frac{c}{3}\left(\left|t_{0}\right|+\Im z\right)\|u\|^{2} \leq\left\|\mathrm{Op}_{h}^{w}\left(z-\tilde{H}_{t_{0}}\right) u\right\|\|u\|+C\left|t_{0}\right|\left\|\mathrm{Op}_{h}^{w}\left(z-\tilde{H}_{t_{0}}\right) u\right\|^{2} \\
\leq \frac{c\left|t_{0}\right|}{6}\|u\|^{2}+\left(\frac{3}{2 c\left|t_{0}\right|}+C\left|t_{0}\right|\right)\left\|\mathrm{Op}_{h}^{w}\left(z-\tilde{H}_{t_{0}}\right) u\right\|^{2}
\end{gathered}
$$

which yields

$$
\frac{c}{6}\left(\left|t_{0}\right|+\Im z\right)\|u\|^{2} \leq\left(\frac{3}{2 c\left|t_{0}\right|}+C\left|t_{0}\right|\right)\left\|\mathrm{Op}_{h}^{w}\left(z-\tilde{H}_{t_{0}}\right) u\right\|^{2},
$$

for all $u \in L^{2}\left(\mathbb{R}^{n} ; \mathbb{C}^{N}\right)$. We conclude that $\left(z-\tilde{H}_{t_{0}}^{w}\right)^{-1}$ extends as a holomorphic function of $z$ to the zone $\Im z \geq-\frac{\left|t_{0}\right|}{2}$. This ends the proof of the lemma.

Let $\tilde{\psi} \in C^{\infty}(\mathbb{R} ; \mathbb{R})$ be such that $\tilde{\psi}(s)=\psi(s)$ for $s>0, \tilde{\psi}(s)=1$ for $-1 / 4 C_{0}<s<0$, and $\tilde{\psi}(s)=0$ for $s<-1 / 2 C_{0}$, and define $\tilde{\psi}_{L}$ as in (2.22). Then we have

$$
\begin{aligned}
\mathcal{I}_{+}(\tau, \varepsilon ; h) & \equiv-\frac{1}{\pi} \int_{\{\Im z>L\}} \bar{\partial}\left(\tilde{f} \psi_{L}\right)(z) \mathcal{F}_{h}^{-1} \theta_{\varepsilon}(\tau-z) \tilde{K}_{t_{0}}(z ; h) L(d z) \\
& \equiv-\frac{1}{\pi} \int_{\{\Im z>0\}} \bar{\partial}\left(\tilde{f} \psi_{L}\right)(z) \mathcal{F}_{h}^{-1} \theta_{\varepsilon}(\tau-z) \tilde{K}_{t_{0}}(z ; h) L(d z) \\
& \equiv-\frac{1}{\pi} \int_{\{\Im z>0\}} \bar{\partial}\left(\tilde{f} \psi_{L} \tilde{\psi}_{L}\right)(z) \mathcal{F}_{h}^{-1} \theta_{\varepsilon}(\tau-z) \tilde{K}_{t_{0}}(z ; h) L(d z) \\
& \equiv \frac{1}{\pi} \int_{\{\Im z<0\}} \bar{\partial}\left(\tilde{f} \psi_{L} \tilde{\psi}_{L}\right)(z) \mathcal{F}_{h}^{-1} \theta_{\varepsilon}(\tau-z) \tilde{K}_{t_{0}}(z ; h) L(d z),
\end{aligned}
$$

uniformly for $\tau \in \mathbb{R}$. Notice that to pass from the first equation to the second we used (2.25), and the last identity follows from the Cauchy formula for analytic functions.

Now, with the same argument as for $\mathcal{I}_{-}$, we see that $\mathcal{I}_{+}=\mathcal{O}\left(\varepsilon^{2} h^{\frac{M}{2}-n-2}\right)$ uniformly for $\tau \in \mathbb{R}$ and $\varepsilon \in\left[h^{1-\delta}, \kappa[\right.$ for all $\kappa>0$, which gives the result since $M>0$ is arbitrary. This ends the proof of Theorem 2.2 .

Remark 3.1. Let $O$ be an open bounded subset of $\mathbb{C}$ such that supp $\tilde{f} \subset O$, and assume that the function $K(z ; h)$ defined by (3.3) in the upper half plane extends as a holomorphic function $\tilde{K}(z ; h)$ to the zone $O_{\ell}(h):=O \cap\{\Im z \geq-\ell \zeta(h)\}$ for all $\ell \in \mathbb{N}$ and that the estimate $\tilde{K}(z ; h)=\mathcal{O}\left(h^{-d(n)}\right)$ holds uniformly for $z \in O_{\ell}(h)$ with $d(n)$ depending only on the dimension. Then (2.3) remains true uniformly for $\varepsilon \in\left[\kappa, h^{-\nu}\right.$ [ with fixed $\kappa>0, \nu \in \mathbb{N}$.

To see this, we first see (3.6), since $\nu$ is fixed and $M$ is arbitrary. Next, since supp $\psi_{L} \subset$ $\left.\left\{z \in \mathbb{C} ;|\Im z| \leq 2 \frac{M}{\kappa} \zeta(h)\right)\right\}$ for all $\varepsilon \in\left[\kappa, h^{-\nu}\right.$, it follows from the above assumption (with $\left.\ell>2 \frac{M}{\kappa}\right)$ and the Cauchy formula that

$$
\begin{aligned}
\mathcal{I}_{+}(\tau, \varepsilon ; h) & =-\frac{1}{\pi} \int_{\{\Im z>0\}} \bar{\partial}\left(\tilde{f} \psi_{L}\right)(z) \mathcal{F}_{h}^{-1} \theta_{\varepsilon}(\tau-z) K(z ; h) L(d z) \\
& =\frac{1}{\pi} \int_{\{\Im z<0\}} \bar{\partial}\left(\tilde{f} \psi_{L}\right)(z) \mathcal{F}_{h}^{-1} \theta_{\varepsilon}(\tau-z) \tilde{K}(z ; h) L(d z) .
\end{aligned}
$$


Then the same argument as for $\mathcal{I}_{-}$shows $\mathcal{I}_{+}=\mathcal{O}\left(h^{\infty}\right)$, and hence (2.3) holds uniformly for $\tau \in \mathbb{R}$ and $\varepsilon \in\left[\kappa, h^{-\nu}[\right.$.

Later, in the application to the study of the SSF, we shall show that assumption (2.15) about the existence of an escape function implies that the function $z \mapsto K(z ; h)$ (defined by (4.1) ) satisfies the condition assumed on $K(z ; h)$ in this remark in an open complex neighborhood $O$ of $\tau_{0}$ (see Lemma 4.2). This will be crucial for the proof of the pointwise asymptotics (2.16).

3.2. Proof of Theorem [2.3, Let $\varepsilon>0$ be a small constant (independent of $h$ ) which will be fixed later. We have

$$
\operatorname{tr}\left(\chi^{w}\left[f\left(H_{j}^{w}\right) \mathcal{F}_{h}^{-1} \theta_{\varepsilon}\left(\tau-H_{j}^{w}\right)\right]_{0}^{1}\right)=I(\tau, \varepsilon ; h)
$$

where $\mathcal{I}(\tau, \varepsilon ; h)$ is defined by (2.23) with

$$
K(z ; h)=\operatorname{tr}\left(\chi^{w}\left[\left(z-H_{j}^{w}\right)^{-1}\right]_{0}^{1}\right) .
$$

It follows from (2.25) and (2.27) that, uniformly for $\tau \in \mathbb{R}$,

$$
\mathcal{I}(\tau, \varepsilon ; h) \equiv-\frac{1}{\pi} \int_{\{L<|\Im z|<2 L\}} \bar{\partial}\left(\tilde{f} \psi_{L}\right)(z) \mathcal{F}_{h}^{-1} \theta_{\varepsilon}(\tau-z) \operatorname{tr}\left(\chi^{w}\left[\left(z-H_{j}^{w}\right)^{-1}\right]_{0}^{1}\right) L(d z) .
$$

Let $B_{0} \in C_{0}^{\infty}\left(\mathbb{R}^{2 n}\right)$ be a real-valued function in the phase space such that $B_{0}=1$ near supp $\chi$ and $B_{0}=0$ near supp $\left(H_{1}-H_{0}\right)$, and let $B=\alpha B_{0}$ for a constant $\alpha>0$ that we will choose later. We notice that the symbol $b=e^{B \log \frac{1}{h}}$ is of class $S_{\delta}^{l}\left(\mathbb{R}^{2 n}\right){ }^{1}$ for $l=\alpha\left\|B_{0}\right\|_{L^{\infty}\left(\mathbb{R}^{2 n}\right)}$ and every $\delta>0$. By the same notation we also denote the corresponding $h$-pseudodifferential operator, which is bounded, elliptic and has an inverse operator $\left(e^{B \log \frac{1}{h}}\right)^{-1}$ with symbol in the same class. Using the $h$-pseudodifferential calculus (see [7, Ch. 7]) as well as the Calderón-Vaillancourt theorem, it is clear that for some $k \in \mathbb{N}$,

$$
e^{B \log \frac{1}{h}}\left(z-H_{1}^{w}\right)\left(e^{B \log \frac{1}{h}}\right)^{-1}=z-H_{1}^{w}+\mathcal{O}(\alpha \zeta(h))\left\|\nabla B_{0}\right\|_{C^{k}},
$$

in operator norm, for $h \leq h(\alpha)$, where $h(\alpha)>0$ is some continuous function.

It follows that for $|\Im z| \geq C \alpha \zeta(h)$ (where $C$ depends only on $\left\|H_{1}\right\|_{C^{k}}$ and $\left\|B_{0}\right\|_{C^{k}}$ ), the right hand side of (3.16) is invertible and we have

$$
\left\|e^{B \log \frac{1}{h}}\left(z-H_{1}^{w}\right)^{-1}\left(e^{B \log \frac{1}{h}}\right)^{-1}\right\|=\mathcal{O}\left(|\Im z|^{-1}\right) .
$$

in operator norm.

On the other hand, since $B=\alpha$ near supp $\chi$ and that $B=0$ near $\operatorname{supp}\left(H_{1}-H_{0}\right)$, it follows from the $h$-pseudodifferential calculus again that we have

$$
\begin{gathered}
\left(e^{B \log \frac{1}{h}}\right)^{-1}\left(H_{1}^{w}-H_{0}^{w}\right)=\left(H_{1}^{w}-H_{0}^{w}\right)+\mathcal{O}\left(h^{\infty}\right), \\
\chi^{w} e^{B \log \frac{1}{h}}=e^{\alpha \log \frac{1}{h}} \chi^{w}+\mathcal{O}\left(h^{\infty}\right),
\end{gathered}
$$

in operator norm and trace norm respectively. Thus

$$
\begin{gathered}
e^{\alpha \log \frac{1}{h}} \operatorname{tr}\left(\chi^{w}\left(z-H_{1}^{w}\right)^{-1}\left(H_{1}^{w}-H_{0}^{w}\right)\left(z-H_{0}^{w}\right)^{-1}\right) \\
=\operatorname{tr}\left(\chi^{w} e^{B \log \frac{1}{h}}\left(z-H_{1}^{w}\right)^{-1}\left(e^{B \log \frac{1}{h}}\right)^{-1}\left(H_{1}^{w}-H_{0}^{w}\right)\left(z-H_{0}^{w}\right)^{-1}\right)+\mathcal{O}\left(\frac{h^{\infty}}{|\Im z|^{2}}\right)
\end{gathered}
$$

\footnotetext{
${ }^{1}$ Following [7], $S_{\delta}^{k}\left(\mathbb{R}^{2 n}\right):=\left\{a(\cdot ; h) \in C^{\infty}\left(\mathbb{R}^{2 n} ; \mathbb{R}\right) ; \forall \alpha \in \mathbb{N}^{2 n}: \partial_{x, \xi}^{\alpha} a(x, \xi ; h)=\mathcal{O}_{\alpha}\left(h^{-\delta|\alpha|-k}\right)\right\}$, for $k \in \mathbb{R}$ and $\delta \in[0,1]$.
} 


$$
=\operatorname{tr}\left(\chi^{w}\left(z-e^{B \log \frac{1}{h}} H_{1}^{w}\left(e^{B \log \frac{1}{h}}\right)^{-1}\right)^{-1}\left(H_{1}^{w}-H_{0}^{w}\right)\left(z-H_{0}^{w}\right)^{-1}\right)+\mathcal{O}\left(\frac{h^{\infty}}{|\Im z|^{2}}\right) .
$$

Combining this with (3.16), we deduce that for $|\Im z| \geq C \alpha \zeta(h)$

$$
\operatorname{tr}\left(\chi^{w}\left[\left(z-H_{j}^{w}\right)^{-1}\right]_{0}^{1}\right)=\mathcal{O}\left(\frac{h^{\alpha-n}}{|\Im z|^{2}}\right) .
$$

We choose $\alpha=\frac{M}{C \varepsilon}$. It follows from (2.28), (3.15) and (3.18)

$$
\mathcal{I}(\tau, \varepsilon ; h)=\mathcal{O}\left(\varepsilon^{3} h^{M\left(\frac{1}{\varepsilon C}-2\right)-n-3} \log \left(\frac{1}{h}\right)^{-2}\right)
$$

Next we choose $\varepsilon$ small enough so that $\frac{1}{\varepsilon C}>2$. This ends the proof of Theorem 2.3 since $M$ is arbitrary. We recall that $C$ depends only on $\left\|H_{1}\right\|_{C^{k}}$ and $\left\|B_{0}\right\|_{C^{k}}$.

3.3. Proof of Theorem 2.4. Without any loss of generality, we may assume that $\chi$ is supported in a small neighborhood of a fixed point $\left(x_{0}, \xi_{0}\right)$. In fact we may replace $\chi$ by a finite sum of terms $\chi \chi_{i}$ with $\sum_{i} \chi_{i}=1$ near the support of $\chi$ and $\chi_{i}$ has its support in a small neighborhood of a fixed point $\left(x_{i}, \xi_{i}\right) \in \operatorname{supp} \chi$. Then, choosing the support of $\chi$ small enough, we may assume that $\tau-H(x, \xi)$ is uniformly microhyperbolic in a fixed direction $T$ for $(x, \xi)$ in supp $\chi$ and for $\tau$ near $\tau_{0}$. Moreover, by modifying $H$ outside supp $\chi$ as in Theorem A.3. we may assume that $\tau-H(x, \xi)$ is uniformly microhyperbolic in the whole phase space $\mathbb{R}^{2 n}$ in the direction $T$ thanks to Theorem 2.3 .

Let $\theta \in C_{0}^{\infty}(]-1 ; 1[; \mathbb{R})$ be equal to one near $0, \varepsilon>0$ small enough independent of $h$ and $D$ be an integer such that $2^{-D} \sim h^{1-\delta}$ with $\left.\delta \in\right] 0, \frac{1}{2}\left[\right.$. Put $\tilde{\varepsilon}=2^{-D} \varepsilon$. We write

$$
\theta_{\varepsilon}(t)-\theta_{\tilde{\varepsilon}}(t)=\sum_{i=1}^{D} \Psi\left(2^{i-1} t\right),
$$

where $\Psi(t)=\theta_{\varepsilon}(t)-\theta_{\varepsilon}(2 t) \in C_{0}^{\infty}(\mathbb{R})$. Clearly, $\Psi(t)=\Psi_{1}(t)+\Psi_{2}(t)$ where $\Psi_{1}$ and $\Psi_{2}$ are equal to 0 near zero, $\left.\operatorname{supp} \Psi_{1} \subset\right] 0, \varepsilon\left[\right.$ and supp $\left.\Psi_{2} \subset\right]-\varepsilon, 0[$. Now applying Theorem 2.2 (resp. Remark 2.1) to $\Psi_{1}\left(2^{i-1} t\right)$ (resp. $\left.\Psi_{2}\left(2^{i-1} t\right)\right), i=1, \cdots D$, we see that there exists $I \in O_{\tau_{0}}$ such that for all $f \in C_{0}^{\infty}(I ; \mathbb{R})$, we have

$$
\operatorname{tr}\left(\chi^{w} f\left(H^{w}\right) \mathcal{F}_{h}^{-1} \theta_{\varepsilon}\left(\tau-H^{w}\right)\right) \equiv \operatorname{tr}\left(\chi^{w} f\left(H^{w}\right) \mathcal{F}_{h}^{-1} \theta_{\tilde{\varepsilon}}\left(\tau-H^{w}\right)\right)
$$

uniformly for $\tau \in \mathbb{R}$. As in (2.26), we have

$$
\begin{gathered}
\operatorname{tr}\left(\chi^{w} f\left(H^{w}\right) \mathcal{F}_{h}^{-1} \theta_{\tilde{\varepsilon}}\left(\tau-H^{w}\right)\right) \equiv \\
-\frac{1}{\pi} \int_{\left\{|\Im z| \geq h^{\delta}\right\}} \bar{\partial}\left(\tilde{f} \psi_{h^{\delta}}\right)(z) \mathcal{F}_{h}^{-1} \theta_{\tilde{\varepsilon}}(\tau-z) \operatorname{tr}\left(\chi^{w}\left(z-H^{w}\right)^{-1}\right) L(d z) .
\end{gathered}
$$

Now in the zone $\Omega_{\delta}:=\left\{z \in \operatorname{supp} \tilde{f} ;|\Im z| \geq h^{\delta}\right\}$, with $0<\delta<\frac{1}{2}$, the resolvent $\left(z-H^{w}\right)^{-1}$ is an $h$-pseudodifferential operator. More precisely, according to Proposition 8.6 in [7], there exists a $C^{\infty}$ matrix-valued function $(x, \xi) \mapsto \mathcal{G}(x, \xi, z ; h)$ such that

$$
\left\|\partial_{x, \xi}^{\alpha} \mathcal{G}(x, \xi, z ; h)\right\| \leq C_{\alpha} h^{-\delta(1+|\alpha|)}, \quad \forall \alpha \in \mathbb{N}^{2 n},
$$

uniformly on $z \in \Omega_{\delta}$ and

$$
\left(z-H^{w}\right)^{-1}=\operatorname{Op}_{h}^{w}(\mathcal{G}(x, \xi, z ; h)),
$$


for all $z \in \Omega_{\delta}$. Moreover

$$
\mathcal{G}(x, \xi, z ; h) \sim \mathcal{G}_{0}(x, \xi, z)+h \mathcal{G}_{1}(x, \xi, z)+h^{2} \mathcal{G}_{2}(x, \xi, z)+\cdots \quad \text { in } S_{\delta}^{\delta}\left(\mathbb{R}^{2 n}, \mathcal{H}_{N}\right)
$$

where $\mathcal{G}_{j}(x, \xi, z)$ is a finite sum of terms of the form

$(z-H(x, \xi))^{-1} B_{1}(x, \xi, z)(z-H(x, \xi))^{-1} B_{2}(x, \xi, z)(z-H(x, \xi))^{-1} \cdots B_{k}(x, \xi, z)(z-H(x, \xi))^{-1}$,

with $k<2 j+1, B_{l}(x, \xi, z) \in S^{0}\left(\mathbb{R}^{2 n} ; \mathcal{H}_{N}\right)$ holomorphic in $z$ near supp $\tilde{f}$. Now by classical results on trace class $h$-pseudodifferential operators (see Theorem II.53 and Proposition II. 56 in [26]), we have for all $m \in \mathbb{N}$,

$$
\operatorname{tr}\left(\chi^{w} f\left(H^{w}\right) \mathcal{F}_{h}^{-1} \theta_{\varepsilon}\left(\tau-H^{w}\right)\right)=(2 \pi h)^{-n} \sum_{j=0}^{m} a_{j}(\tau ; h) h^{j}+\mathcal{O}\left(h^{(m+1)(1-2 \delta)-n}\right),
$$

where

$$
a_{j}(\tau ; h)=-\frac{1}{\pi} \int \bar{\partial}\left(\tilde{f} \psi_{h^{\delta}}\right)(z) \mathcal{F}_{h}^{-1} \theta_{\tilde{\varepsilon}}(\tau-z) \widehat{e}_{j}(z) L(d z)
$$

with

$$
\widehat{e}_{j}(z):=\iint_{\mathbb{R}^{2 n}} \chi(x, \xi) \widehat{\operatorname{tr}}\left(\mathcal{G}_{j}(x, \xi, z)\right) d x d \xi .
$$

Here $\widehat{\operatorname{tr}}$ denotes the trace of square matrices. The microhyperbolicity assumption implies that there exists $I \in O_{\tau_{0}}$ such that the function

$$
I \ni \tau \mapsto \widehat{e}_{j}(\tau \pm i 0):=\lim _{s \searrow 0} \widehat{e}_{j}(\tau \pm i s),
$$

is $C^{\infty}$ (see Proposition A.4. Set

$$
\gamma_{j}(\tau):=-\frac{1}{2 \pi i}\left[\widehat{e}_{j}(\tau+i 0)-\widehat{e}_{j}(\tau-i 0)\right]
$$

Now the following lemma ends the proof of Theorem 2.4.

\section{Lemma 3.2.}

$$
a_{j}(\tau ; h)=f(\tau) \gamma_{j}(\tau)+\mathcal{O}\left(h^{\infty}\right) .
$$

Proof. Since $z \mapsto \mathcal{F}_{h}^{-1} \theta_{\tilde{\varepsilon}}(\tau-z) \widehat{e}_{j}(z)$ is holomorphic in the complex domain $\{z \in \mathbb{C} ; \pm \Im z>0\}$, it follows from the Green formula that

$$
\begin{gathered}
a_{j}(\tau ; h)=-\frac{1}{\pi} \lim _{s \searrow 0} \int_{\{\Im z>s\}} \bar{\partial}\left(\tilde{f} \psi_{h^{\delta}}\right)(z) \mathcal{F}_{h}^{-1} \theta_{\tilde{\varepsilon}}(\tau-z) \widehat{e}_{j}(z) L(d z) \\
-\frac{1}{\pi} \lim _{s \searrow 0} \int_{\{\Im z<-s\}} \bar{\partial}\left(\tilde{f} \psi_{h^{\delta}}\right)(z) \mathcal{F}_{h}^{-1} \theta_{\tilde{\varepsilon}}(\tau-z) \widehat{e}_{j}(z) L(d z) \\
=-\frac{1}{2 \pi i} \int_{\mathbb{R}} f(\lambda) \mathcal{F}_{h}^{-1} \theta_{\tilde{\varepsilon}}(\tau-\lambda)\left[\widehat{e}_{j}(\tau+i 0)-\widehat{e}_{j}(\tau-i 0)\right] d \lambda \\
=\int_{\mathbb{R}} \mathcal{F}_{h}^{-1} \theta_{\tilde{\varepsilon}}(\tau-\lambda) f(\lambda) \gamma_{j}(\lambda) d \lambda=\int_{\mathbb{R}} \mathcal{F}_{1}^{-1} \theta(\lambda) f\left(\tau-h^{\delta} \lambda\right) \gamma_{j}\left(\tau-h^{\delta} \lambda\right) d \lambda
\end{gathered}
$$

The last equality is obtained by a change of variable. Applying Taylor's formula to the function $\lambda \mapsto f\left(\tau-h^{\delta} \lambda\right) \gamma_{j}\left(\tau-h^{\delta} \lambda\right)$ at $\lambda=0$ and using the fact that $\int \mathcal{F}_{1}^{-1} \theta(\lambda)(-i \lambda)^{k} d \lambda=$ $\theta^{(k)}(0)=0$ we obtain the lemma. We recall that $\theta=1$ near zero. Here $\mathcal{F}_{1}^{-1}$ is $\mathcal{F}_{h}^{-1}$ with $h=1$ 


\section{Proofs of the Results on the SSF}

This section is devoted to the proofs of the results of subsection 2.2 .

We follow the notations used in section 3. From the assumption (2.7), the operator

$$
\left[\left(P_{j}(h)-z_{0}\right)^{-q}\left(z-P_{j}(h)\right)^{-1}\right]_{0}^{1}
$$

is of trace class for $q>\frac{n}{2}$ and $z_{0} \notin \sigma\left(P_{1}(h)\right) \cup \sigma\left(P_{0}(h)\right)$ which are fixed in what follows.

We set

$$
K(z ; h):=\left(z-z_{0}\right)^{q} \operatorname{tr}\left(\left[\left(P_{j}(h)-z_{0}\right)^{-q}\left(z-P_{j}(h)\right)^{-1}\right]_{0}^{1}\right), \quad \Im z \neq 0 .
$$

As in the proof of (3.4), formulas (2.8) and (3.2) yield, for all $f, \theta \in C_{0}^{\infty}(\mathbb{R} ; \mathbb{R})$,

$$
\begin{aligned}
\left\langle s_{h}^{\prime}(\cdot), f(\cdot)\right\rangle & =\frac{1}{\pi} \int_{\mathbb{C}} \bar{\partial} \tilde{f}(z) K(z ; h) L(d z), \\
\left\langle s_{h}^{\prime}(\cdot), \mathcal{F}_{h}^{-1} \theta(\tau-\cdot) f(\cdot)\right\rangle & =\frac{1}{\pi} \int_{\mathbb{C}} \bar{\partial} \tilde{f}(z) \mathcal{F}_{h}^{-1} \theta(\tau-z) K(z ; h) L(d z) .
\end{aligned}
$$

4.1. Proof of Theorem 2.5. This is a classical result and follows from the functional calculus of $h$-pseudodifferential operators.

Let $\delta \in] 0, \frac{1}{2}\left[\right.$. The contribution from the domain $|\operatorname{Im} z| \leq h^{\delta}$ of the integral in the right hand side of (4.2) is $\mathcal{O}\left(h^{\infty}\right)$.

Next, in the domain $|\operatorname{Im} z| \geq h^{\delta}$, we use the fact that $\left(z-P_{k}(h)\right)^{-1}$ are $h$-pseudodifferential operators, $k=0,1$ (see (3.21) and (3.22)). This formally yields (2.9) (with $q=0$ ) with

$$
c_{j}(f)=\iint_{\mathbb{R}^{2 n}} \frac{1}{\pi} \widehat{\operatorname{tr}}\left(\int_{\mathbb{C}} \bar{\partial} \tilde{f}(z)\left(\mathcal{G}_{j, 1}(x, \xi, z)-\mathcal{G}_{j, 0}(x, \xi, z)\right) L(d z)\right) d x d \xi .
$$

In particular

$$
c_{0}(f)=\iint_{\mathbb{R}^{2 n}} \widehat{\operatorname{tr}}\left(f\left(p_{0}(x, \xi)\right)-f\left(p_{1}(x, \xi)\right)\right) d x d \xi,
$$

and (2.10) trivially follows from this formula. To see that $c_{2 j+1}(f)=0$, it suffices to notice that $h \mapsto|2 \pi h|^{n} \operatorname{tr}\left(f\left(P_{1}(h)\right)-f\left(P_{0}(h)\right)\right.$ is an even function. More rigorously for $q \neq 0$, one may write $K(z ; h)$ as

$$
\begin{aligned}
K(z ; h) & =\left(z-z_{0}\right)^{q} \operatorname{tr}\left[\left(\left(P_{1}(h)-z_{0}\right)^{-q}-\left(P_{0}(h)-z_{0}\right)^{-q}\right)\left(z-P_{1}(h)\right)^{-1}\right] \\
& +\left(z-z_{0}\right)^{q} \operatorname{tr}\left[\left(P_{0}(h)-z_{0}\right)^{-q}\left(\left(z-P_{1}(h)\right)^{-1}-\left(z-P_{0}(h)\right)^{-1}\right)\right],
\end{aligned}
$$

and use the fact that $\left(P_{k}(h)-z_{0}\right)^{-q}$ are $h$-pseudodifferential operators, $k=0,1$. This ends the proof of (2.9) .

4.2. Proof of Theorem 2.6. The proof of Theorem 2.6 uses (4.3) and is quite similar to that of Theorem 2.4, and we omit the details. The main difference is that $\Sigma_{\tau_{0}}=\{(x, \xi) \in$ $\left.\mathbb{R}^{2 n} ; \operatorname{det}\left(p_{1}(x, \xi)-\tau_{0}\right)=0\right\}$ is not a compact set in $\mathbb{R}^{2 n}$. In this case we have to justify that we can cover $\Sigma_{\tau_{0}}$ by finite open sets $O_{1}, O_{2}, \cdots, O_{\ell}$ in which we can construct $\tilde{p}_{1, k}(x, \xi)$, and $T_{k} \in \mathbb{R}^{2 n}, k=1, \cdots, \ell$, such that $\tilde{p}_{1, k}(x, \xi)-\tau_{0}$ is uniformly microhyperbolic in the direction $T_{k}$ and $\tilde{p}_{1, k}(x, \xi)=p_{1}(x, \xi)$ for all $(x, \xi) \in O_{k}$. To see this, we first notice that $\Sigma_{\tau_{0}}=\Sigma_{\tau_{0}} \cap\left\{|\xi| \leq R_{0}\right\}$ (R being large enough), since $\lim _{|\xi| \rightarrow \infty} \operatorname{det}\left(p_{1}(x, \xi)-\tau_{0}\right)=\infty$. Next, 
fix $R_{1}$ large such that $\inf _{|x|>R_{1}}\left|\operatorname{det}\left(V(x)-\tau_{0}\right)\right|>0$. This is possible since $\lim _{|x| \rightarrow \infty} V(x)=V_{\infty}$ and $\tau_{0} \notin \sigma\left(V_{\infty}\right)$ by assumption. On the compact set $\Sigma_{\tau_{0}} \cap\left\{|x| \leq R_{1}\right\}$ we can apply Theorem 2.4 without any modification. On the other hand, we see from the choice of $R_{1}$ that $\nabla\left(|\xi|^{2}\right) \neq 0$ for all $(x, \xi) \in \Sigma_{\tau_{0}} \cap\left\{|x|>R_{1}\right\}:=\Sigma_{\tau_{0}, R_{1}}$. Thus, we can find finite open covers $o_{1}, o_{2}, \cdots o_{\ell}$ in $\mathbb{R}^{n}, \tilde{T}_{1}, \tilde{T}_{2}, \cdots, \tilde{T}_{\ell} \in \mathbb{R}^{n}$ and $c_{1}, \cdots c_{\ell}>0$ such that $\left\{|\xi| \leq R_{0}\right\} \subset \bigcup_{j=1}^{\ell} o_{j}$ and for each $j=1, \cdots \ell,\left\langle\tilde{T}_{j}, \nabla\left(|\xi|^{2}\right)\right\rangle \geq c_{j}$, uniformly on $\xi \in o_{j} \cap \pi_{\xi} \Sigma_{\tau_{0}, R_{1}}$. Now using Theorem A.3. we construct $\tilde{p}_{1, j}(x, \xi)$, such that $\tilde{p}_{1, j}(x, \xi)-\tau_{0}$ is uniformly microhyperbolic in the direction $T_{j}=\left(0, \tilde{T}_{j}\right)$ and $\tilde{p}_{1, j}(x, \xi)=p_{1}(x, \xi)$ for all $(x, \xi) \in\left\{|x|>R_{1}\right\} \times o_{j}$. We can now proceed analogously to the proof of Theorem 2.4 .

4.3. Proof of Theorem 2.7. For the proof of Theorem 2.7. assume that $\tau \mapsto s(\tau ; h)$ is monotonic (i.e., $s^{\prime}(\cdot ; h)$ is positive or negative in the sense of distributions). In this case Theorem 2.7 is a simple consequence of Theorem 2.6 by standard Tauberian arguments (see [7], 14], [26]).

For the general case, we use a trick due to Robert [27, which consists in writing $s(\tau ; h)=$ $s_{1}(\tau ; h)-s_{2}(\tau ; h)$ where $\tau \mapsto s_{i}(\tau ; h), i=1,2$ are monotonic. Now, it suffices to apply the above argument to each $s_{i}(\tau ; h)$.

Notice that, Robert's trick applies to Schrödinger operators with matrix-valued potential under the assumption (2.7) with scalar matrix $V_{\infty}$.

4.4. Proof of Theorem 2.8. The proof of the following lemma is the same as that of Lemma 2.2 in $[8$.

Lemma 4.1. Under the assumption (2.7), we have

$$
s_{h}^{\prime}(\tau)=\frac{1}{\pi} \Im K(\tau+i 0 ; h) \text { in } \mathcal{D}^{\prime}(\mathbb{R}),
$$

i.e. we have, for all $f \in C_{0}^{\infty}(\mathbb{R})$,

$$
\left\langle s_{h}^{\prime}(\cdot), f\right\rangle=\lim _{\kappa \searrow 0} \frac{1}{\pi} \int_{\mathbb{R}} f(\tau) \Im K(\tau+i \kappa ; h) d \tau .
$$

Let $I \in O_{\tau_{0}}$ such that (2.15) holds on $\Sigma_{\tau}$ for all $\tau \in I$. For $M \geq 0$, we introduce the following $h$-dependent set

$$
\Gamma_{M}:=\{z \in \mathbb{C} ; \Re z \in I \text { and } \Im z>-M \zeta(h)\},
$$

where we recall that $\zeta(h)=h \log \left(\frac{1}{h}\right)$.

The idea of the proof of the following lemma is based on the theory of resonance and close to the one of Theorem 1 in [32].

Lemma 4.2. In addition to the assumptions (2.7) and (2.15), we assume that $V(x)-V_{\infty} \in$ $C_{0}^{\infty}\left(\mathbb{R}^{n} ; \mathcal{H}_{N}\right)$. For any $M>0$, the function $z \mapsto K(z ; h)$ has an analytic extension from $\Gamma_{0}$ to $\Gamma_{M}$. Moreover, we have, uniformly for $z \in \Gamma_{M}$,

$$
K^{(k)}(z ; h)=\mathcal{O}\left(h^{-n} \zeta(h)^{-k-1}\right), \quad \forall k \in \mathbb{N} .
$$

In particular, uniformly for $\tau \in I$,

$$
s_{h}^{(k+1)}(\tau)=\mathcal{O}\left(h^{-n} \zeta(h)^{-k-1}\right), \quad \forall k \in \mathbb{N} .
$$


Proof. The estimate (4.7) follows immediately from (4.6) and the representation of the SSF given by Lemma 4.1. Hence it is enough to prove (4.6).

Let $F: \mathbb{R}^{n} \rightarrow \mathbb{R}^{n}$ be a smooth vector field such that $F=0$ in a neighbourhood of $\operatorname{supp}\left(V-V_{\infty}\right)$ and $F(x)=x$ for $|x|$ large enough. For $\omega \in \mathbb{R}$ small enough, we denote $U_{\omega}: L^{2}\left(\mathbb{R}^{n} ; \mathbb{C}^{N}\right) \rightarrow L^{2}\left(\mathbb{R}^{n}, \mathbb{C}^{N}\right)$ the unitary operator defined by

$$
U_{\omega} \phi(x):=|\operatorname{det}(1+\omega \nabla F(x))|^{\frac{1}{2}} \phi(x+\omega F(x)),
$$

and set

$$
P_{j, \omega}(h):=U_{\omega} P_{j}(h)\left(U_{\omega}\right)^{-1}, \quad j=0,1 .
$$

They are differential operators with analytic coefficients with respect to $\omega$, and can be analytically continued to small enough complex values of $\omega$. It follows from the analytic perturbation theory (see [16]) that for $\omega_{0}$ small enough, $\left.\omega \in\right]-\omega_{0}, \omega_{0}\left[\mapsto P_{j, \omega}(h), j=0,1\right.$, extends to an analytic type $\mathcal{A}$-family of operators on $D\left(\omega_{0}\right):=\left\{\omega \in \mathbb{C} ;|\omega|<\omega_{0}\right\}$ with domain $H^{2}\left(\mathbb{R}^{n} ; \mathbb{C}^{N}\right)$.

We set, first for real $\omega$ and $\Im z>0$,

$$
K_{\omega}(z ; h):=\left(z-z_{0}\right)^{q} \operatorname{tr}\left(\left[\left(P_{j, \omega}(h)-z_{0}\right)^{-q}\left(z-P_{j, \omega}(h)\right)^{-1}\right]_{0}^{1}\right) .
$$

Since $U_{\omega}$ is unitary for real $\omega$, it follows from the cyclicity of the trace that

$$
\left.K(z ; h)=K_{\omega}(z ; h), \quad \forall \omega \in\right]-\omega_{0}, \omega_{0}[, \forall \Im z>0 .
$$

On the other hand, for $\Im z>M_{0} \zeta(h)$ for a given $h$-independent $M_{0}>0$, the function $\omega \mapsto K_{\omega}(z)$ is analytic in $\omega \in D\left(2 c M_{0} \zeta(h)\right)$ with some $c>0$ independent of $h$ and $M_{0}$. Thus, by the uniqueness theorem of analytic continuation, the identity (4.10) remains true for $\Im z>M_{0} \zeta(h)$ and $\omega \in D\left(2 c M_{0} \zeta(h)\right)$, i.e.,

$$
K(z ; h)=K_{\omega}(z ; h), \quad \forall \omega \in D\left(2 c M_{0} \zeta(h)\right), \forall \Im z>M_{0} \zeta(h) .
$$

From now on we fix $M=c M_{0}$ and set $\omega_{1}=i M \zeta(h)$.

Since $\tau_{0}>e_{N, \infty}, x \cdot \xi$ is an escape function for $p_{1}(x, \xi)$ for $|(x, \xi)|$ large enough. Thus, without any loss of generality, we may assume that $G(x, \xi)=x \cdot \xi$ for $|(x, \xi)|$ large enough. Then $\tilde{G}(x, \xi):=G(x, \xi)-F(x) \cdot \xi$ has a compact support, and in particular its quantization $\tilde{G}^{w}\left(x, h D_{x}\right)$ is $L^{2}$-bounded by the Calderón-Vaillancourt theorem and the operators $e^{ \pm \frac{M \zeta(h)}{h}} \tilde{G}^{w}\left(x, h D_{x}\right)$ are well-defined.

Let us define

$$
\begin{gathered}
\tilde{P}_{j, \omega_{1}}(h):=e^{-\frac{M \zeta(h)}{h} \tilde{G}^{w}\left(x, h D_{x}\right)} P_{j, \omega_{1}}(h) e^{\frac{M \zeta(h)}{h} \tilde{G}^{w}\left(x, h D_{x}\right)}, \quad j=0,1, \\
\tilde{K}_{\omega_{1}}(z ; h):=\left(z-z_{0}\right)^{q} \operatorname{tr}\left(\left[\left(\tilde{P}_{j, \omega_{1}}(h)-z_{0}\right)^{-q}\left(z-\tilde{P}_{j, \omega_{1}}(h)\right)^{-1}\right]_{0}^{1}\right) .
\end{gathered}
$$

From Lemma 4.3 below, $z \mapsto \tilde{K}_{\omega_{1}}(z ; h)$ is analytic in $\Gamma_{c M}$ for some $c>0$. Again by the cyclicity of the trace and the uniqueness of the analytic continuation, we conclude

$$
\tilde{K}_{\omega_{1}}(z ; h)=K(z ; h), \quad \forall z \in \Gamma_{c M} .
$$

This with the resolvent estimate (4.14) leads to

$$
K(z ; h)=\mathcal{O}\left(h^{-n} \zeta(h)^{-1}\right),
$$


uniformly for $z \in \Gamma_{c M}$, which yields (4.6) for $k=0$. Next, taking the derivative of (4.12) and applying (4.14) we obtain (4.6) for $k \geq 1$ (Recall that the trace of semiclassical quantization of a symbol in a suitable class is of $\mathcal{O}\left(h^{-n}\right)$, see [7, Theorem 9.4]).

Lemma 4.3. There exists $c>0$ such that for all $M>0$ the operator $\tilde{P}_{j, \omega_{1}}(h)-z$ is invertible for every $z \in \Gamma_{c M}$. Moreover, one has, uniformly in this domain,

$$
\left\|\left(z-\tilde{P}_{j, \omega_{1}}(h)\right)^{-1}\right\|=\mathcal{O}\left(\zeta(h)^{-1}\right) .
$$

Proof. We have

$$
\tilde{P}_{j, \omega_{1}}(h)=e^{-\frac{M \zeta(h)}{h} \operatorname{ad}_{\tilde{G}^{w}}} P_{j, \omega_{1}}(h) \sim \sum_{k=0}^{\infty} \frac{(-M \zeta(h))^{k}}{k !}\left(\frac{1}{h} \operatorname{ad}_{\tilde{G}^{w}}\right)^{k} P_{j, \omega_{1}}(h),
$$

where $\operatorname{ad}_{\tilde{G}^{w}} P_{j, \omega_{1}}(h)=\left[\tilde{G}^{w}, P_{j, \omega_{1}}(h)\right]=\mathcal{O}(h)$ 2. By definition, $\zeta(h)$ tends to 0 as $h \searrow 0$. Combining this with the boundedness of $h^{-1} \mathrm{ad}_{\tilde{G}}$ we find that the asymptotic expansion (4.15) makes sense. In particular,

$$
\tilde{P}_{j, \omega_{1}}(h)=P_{j, \omega_{1}}(h)-\frac{M \zeta(h)}{h}\left[\tilde{G}^{w}\left(x, h D_{x}\right), P_{j, \omega_{1}}(h)\right]+\mathcal{O}\left(M^{2} \zeta(h)^{2}\right) .
$$

Let $p_{j, \omega_{1}}, \tilde{p}_{j, \omega_{1}}$ be the Weyl symbols of $P_{j, \omega_{1}}, \tilde{P}_{j, \omega_{1}}$ respectively. We obtain from the $h$ pseudodifferential calculus ([7, Ch. 7]),

$$
\tilde{p}_{j, \omega_{1}}=p_{j, \omega_{1}}-i M \zeta(h)\left\{p_{j, \omega_{1}}, \tilde{G}\right\}+\mathcal{O}\left(M^{2} \zeta(h)^{2}\right),
$$

and in particular, using the Taylor expansion of $p_{j, \omega_{1}}$ with respect to $\omega_{1}$;

$$
p_{j, \omega_{1}}=p_{j}-i M \zeta(h)\left\{p_{j}, F(x) \cdot \xi\right\}+\mathcal{O}\left(M^{2} \zeta(h)^{2}\right),
$$

we obtain

$$
\begin{gathered}
\Im\left(\tilde{p}_{j, \omega_{1}}\right)=-M \zeta(h)\left\{p_{j}, \tilde{G}+F(x) \cdot \xi\right\}+\mathcal{O}\left(M^{2} \zeta(h)^{2}\right) . \\
\Re\left(\tilde{p}_{j, \omega_{1}}\right)=p_{j}+\mathcal{O}(M \zeta(h)) .
\end{gathered}
$$

Since $G(x, \xi)=\tilde{G}(x, \xi)+F(x) \cdot \xi$ satisfies the assumption (2.15), it follows from (4.17) and (4.18) that there exist $C>0$ and $I \in O_{\tau_{0}}$ such that

$$
-\Im\left(\tilde{p}_{1, \omega_{1}}\right)(x, \xi) \geq C M \zeta(h), \quad \forall(x, \xi) \in \Sigma_{I}:=\bigcup_{\tau \in I} \Sigma_{\tau},
$$

Of course, the same estimate holds also for $\Im\left(\tilde{p}_{0, \omega_{1}}\right)(x, \xi)$, since (2.15) always holds for $p_{0}$ with $G=x \cdot \xi$ for any $\tau_{0}>e_{N, \infty}$.

We write $\tilde{P}_{j, \omega_{1}}(h)-z=A_{j, \omega_{1}}(h)-\Re z+i\left(B_{j, \omega_{1}}(h)-\Im z\right)$ with

$$
A_{j, \omega_{1}}(h)=\frac{1}{2}\left(\tilde{P}_{j, \omega_{1}}(h)+\left(\tilde{P}_{j, \omega_{1}}(h)\right)^{*}\right), \quad B_{j, \omega_{1}}(h)=\frac{1}{2 i}\left(\tilde{P}_{j, \omega_{1}}(h)-\left(\tilde{P}_{j, \omega_{1}}(h)\right)^{*}\right) .
$$

Let $\psi_{j, 1}, \psi_{j, 2} \in C^{\infty}\left(\mathbb{R}^{2 n} ; \mathbb{R}\right)$ be such that, for $I^{\prime} \Subset I$,

$$
\psi_{j, 1}^{2}+\psi_{j, 2}^{2}=1, \quad \psi_{j, 1}=1 \text { on } \Sigma_{I^{\prime}}^{j}, \quad \operatorname{supp}\left(\psi_{j, 1}\right) \subset \Sigma_{I}^{j} .
$$

\footnotetext{
${ }^{2}$ We have used the fact $G$ is scalar valued only to prove that $\left[\tilde{G}^{w}, P_{j, \omega_{1}}(h)\right]=\mathcal{O}(h)$.
} 
According to Lemma 3.2 in [32], there exist two self-adjoint operators $\Psi_{j, 1}$ and $\Psi_{j, 2}$ with principal symbols respectively $\psi_{j, 1}$ and $\psi_{j, 2}$ such that

$$
\left(\Psi_{j, 1}\right)^{2}+\left(\Psi_{j, 2}\right)^{2}=\mathrm{Id}+\mathcal{O}\left(h^{\infty}\right) \quad \text { in } \mathcal{L}\left(L^{2}\left(\mathbb{R}^{n}\right)\right) .
$$

We denote by the same letters the operators $\Psi_{j, i}:=\Psi_{j, i} I_{N}, i=1,2$. On the support of $\psi_{j, 1}$, we see from (4.19) that the principal symbol of $-B_{j, \omega_{1}}(h)$ is estimated from below by $C M \zeta(h)$. Then by the Gårding's inequality, we obtain, uniformly for $\Im z>-\frac{C}{3} M \zeta(h)$,

$$
\begin{gathered}
\left\|\left(\tilde{P}_{j, \omega_{1}}(h)-z\right) \Psi_{j, 1} u\right\| \cdot\left\|\Psi_{j, 1} u\right\| \geq\left|\left\langle\left(\tilde{P}_{j, \omega_{1}}(h)-z\right) \Psi_{j, 1} u, \Psi_{j, 1} u\right\rangle\right| \\
\geq\left|\left\langle\left(\Im \tilde{P}_{j, \omega_{1}}(h)-\Im z\right) \Psi_{j, 1} u, \Psi_{j, 1} u\right\rangle\right|=\left\langle\left(\Im z-B_{j, \omega_{1}}(h)\right) \Psi_{j, 1} u, \Psi_{j, 1} u\right\rangle \\
\geq(\Im z+C M \zeta(h)-\mathcal{O}(h))\left\|\Psi_{j, 1} u\right\|^{2} \geq \frac{C}{3} M \zeta(h)\left\|\Psi_{j, 1} u\right\|^{2} .
\end{gathered}
$$

On the other hand, since $A_{j, \omega_{1}}(h)-\Re z$ is uniformly elliptic on the support of $\psi_{j, 2}$ and $\Re z \in$ $I$, the symbolic calculus permits us to construct a parametrix $R \in S^{0}\left(\langle\xi\rangle^{-2}\right)$ of $A_{j, \omega_{1}}(h)-\Re z$ such that, in the symbol sense,

$$
R \#\left(A_{j, \omega_{1}}(h)-\Re z\right) \psi_{j, 2}=\psi_{j, 2}+\mathcal{O}\left(h^{\infty}\right),
$$

where \# stands for the Weyl composition of symbols. As a consequence, we obtain

$$
\left\|\left(\tilde{P}_{j, \omega_{1}}(h)-z\right) \Psi_{j, 2} u\right\| \geq \frac{1}{C^{\prime}}\left\|\Psi_{j, 2} u\right\|-\mathcal{O}\left(h^{\infty}\right)\|u\|^{2} .
$$

Furthermore, by means of standard elliptic arguments, one can easily prove the following semiclassical inequality

$$
\left\|\left[\tilde{P}_{j, \omega_{1}}(h), \Psi_{j, i}\right] u\right\| \leq C_{2} h\left(\left\|\tilde{P}_{j, \omega_{1}}(h) u\right\|+\|u\|\right), \quad \forall u \in H^{2}\left(\mathbb{R}^{n} ; \mathbb{C}^{N}\right) .
$$

Combining (4.20), (4.21), (4.22), and (4.23) with the estimate

$$
\begin{gathered}
\left\|\left(\tilde{P}_{j, \omega_{1}}(h)-z\right) u\right\|^{2}=\sum_{i=1}^{2}\left\|\Psi_{j, i}\left(\tilde{P}_{j, \omega_{1}}(h)-z\right) u\right\|^{2}-\mathcal{O}\left(h^{\infty}\right)\left\|\left(\tilde{P}_{j, \omega_{1}}(h)-z\right) u\right\|^{2} \\
\geq \frac{1}{2} \sum_{i=1}^{2}\left\|\left(\tilde{P}_{j, \omega_{1}}(h)-z\right) \Psi_{j, i} u\right\|^{2}-\sum_{i=1}^{2}\left\|\left[\tilde{P}_{j, \omega_{1}}(h), \Psi_{j, i}\right] u\right\|^{2}-\mathcal{O}\left(h^{\infty}\right)\left\|\left(\tilde{P}_{j, \omega_{1}}(h)-z\right) u\right\|^{2},
\end{gathered}
$$

we deduce, for $z \in \Gamma_{c M}$ (with $c>0$ independent of $M$ and $h$ ) and sufficiently small $h$,

$$
\left\|\left(\tilde{P}_{j, \omega_{1}}(h)-z\right) u\right\| \geq \frac{\zeta(h)}{C}\|u\| .
$$

By the same arguments, we prove an estimate similar to 4.25) for the adjoint operator $\tilde{P}_{j, \omega_{1}}(h)^{*}-\bar{z}$ and we conclude that $\tilde{P}_{j, \omega_{1}}(h)-z$ is invertible for every $z \in \Gamma_{c M}$. Moreover (4.25) yields the resolvent estimate (4.14).

\section{End of the proof of Theorem 2.8 .}

Using Lemma 4.2, and applying Theorem 2.2 and Remark 3.1 to the right hand side of (4.3) we obtain the following lemma. 
Lemma 4.4. Assume that $\varphi \in C_{0}^{\infty}(]-1,1[; \mathbb{R})$ is 0 in a neighborhood of 0 . Let $\kappa$ be a positive constant independent of $h$ and $\nu \in \mathbb{N}$. Under the assumptions of Lemma 4.1, there exists $I \in O_{\tau_{0}}$ such that for $f \in C_{0}^{\infty}(I ; \mathbb{R})$, we have

$$
\left\langle s_{h}^{\prime}(\cdot), \mathcal{F}_{h}^{-1} \varphi_{\varepsilon}(\tau-\cdot) f(\cdot)\right\rangle=\mathcal{O}\left(h^{\infty}\right),
$$

uniformly for $\tau \in \mathbb{R}$ and $\varepsilon \in] \kappa, h^{-\nu}[$.

Now let $\theta \in C_{0}^{\infty}(]-1,1[; \mathbb{R})$ be equal to one near 0 and let $f \in C_{0}^{\infty}(I ; \mathbb{R})$ be as in the above lemma. Suppose $\varepsilon>0$ is a small enough constant independent of $h$ and $\tilde{\varepsilon}:=h^{-\nu}$ with $\nu \in \mathbb{N}$ arbitrary large.

Repeating the same construction as in the proof of Theorem 2.4, we represent the difference $\theta_{\tilde{\varepsilon}}-\theta_{\varepsilon}$ as a finite $\operatorname{sum} \sum_{0 \leq j \leq N(h)} \varphi_{\varepsilon_{j}}$ with $\varphi_{\varepsilon_{j}}$ as in Lemma 4.4 and $N(h)=\mathcal{O}\left(h^{-\nu}\right)$. Applying Lemma 4.4 to each term, we get

$$
\left\langle s_{h}^{\prime}(\cdot), \mathcal{F}_{h}^{-1} \theta_{\varepsilon}(\tau-\cdot) f(\cdot)\right\rangle=\left\langle s_{h}^{\prime}(\cdot), \mathcal{F}_{h}^{-1} \theta_{\tilde{\varepsilon}}(\tau-\cdot) f(\cdot)\right\rangle+\mathcal{O}\left(h^{\infty}\right),
$$

uniformly with respect to $\tau \in \mathbb{R}$.

Next, by a change of variable we have

$$
\left\langle s_{h}^{\prime}(\cdot), \mathcal{F}_{h}^{-1} \theta_{\tilde{\varepsilon}}(\tau-\cdot) f(\cdot)\right\rangle=\int_{\mathbb{R}} \mathcal{F}_{1}^{-1} \theta(\lambda)\left(f s_{h}^{\prime}\right)\left(\tau-h^{1+\nu} \lambda\right) d \lambda .
$$

Applying Taylor's formula to the function $\lambda \mapsto\left(f s_{h}^{\prime}\right)\left(\tau-h^{1+\nu} \lambda\right)$ at $\lambda=0$ and using (4.7) with $k=1$, we get

$$
\left\langle s_{h}^{\prime}(\cdot), \mathcal{F}_{h}^{-1} \theta_{\tilde{\varepsilon}}(\tau-\cdot) f(\cdot)\right\rangle=s_{h}^{\prime}(\tau) f(\tau)+\mathcal{O}\left(h^{\nu+1-n} \zeta(h)^{-2}\right),
$$

uniformly for $\tau \in \mathbb{R}$ since $\int_{\mathbb{R}} \mathcal{F}_{1}^{-1} \theta(\lambda) d \lambda=\theta(0)=1$.

From (4.27) and (4.28) we deduce

$$
s_{h}^{\prime}(\tau) f(\tau)=\left\langle s_{h}^{\prime}(\cdot), \mathcal{F}_{h}^{-1} \theta_{\epsilon}(\tau-\cdot) f(\cdot)\right\rangle+\mathcal{O}\left(h^{\nu+1-n} \zeta(h)^{-2}\right) .
$$

By Theorem 2.6, the first term of the right hand side of the above equality has an asymptotic expansion in powers of $h$. Now, since $\nu$ is arbitrary, the asymptotic expansion (2.16) follows from (4.29) by choosing $f$ equal to 1 near $\tau_{0}$. This ends the proof of Theorem 2.8 under the assumption $V-V_{\infty} \in C_{0}^{\infty}\left(\mathbb{R}^{n} ; \mathcal{H}_{N}\right)$.

Remark 4.1. Notice that, except for Lemma 4.2, all the steps of the proof of Theorem 2.8 remain valid under the assumptions (2.7) and (2.15) with $\mu>n$. We will now show how to dispense with the assumption on the support of $V$ in Lemma 4.2. According to Proposition 4.2 in [24], if $V$ satisfies (2.7), then for any $\varkappa>0$ and $\tilde{\mu} \in] 0, \mu\left[\right.$, we can construct $V_{\varkappa}$ such that $V_{\varkappa}$ can be extended into a holomorphic function of $r=|x|$ in the sector $\Sigma(2 \varkappa)=\{\Re r \geq$ $1 ;|\Im r|<2 \varkappa \Re r\}$, and, for any multi-index $\alpha$, it satisfies

$$
\left\|\partial_{x}^{\alpha}\left(V_{\varkappa}(x)-V(x)\right)\right\|_{N \times N}=\mathcal{O}\left(\langle x\rangle^{-\tilde{\mu}-|\alpha|} \varkappa^{\infty}\right) .
$$

As in [24], we fix $\varkappa=h^{s}$ with $\left.s \in\right] 0,1\left[\right.$. We denote by $K_{\varkappa}(z ; h)$ the right hand side of (4.1) when we replace $V$ by $V_{\varkappa}$ in $P_{1}(h)$. The operator $P_{1}(h)=-h^{2} \Delta+V_{\varkappa}$ can be distorded analytically into $\tilde{P}_{1}(h)=U_{\nu} P_{1}(h)\left(U_{\nu}\right)^{-1}$ (see [24]). Now the proof of Lemma 4.2 shows that (4.6) and (4.7) hold for $K_{\varkappa}(z ; h)$. On the other hand, using the resolvent identity and we show that

$$
K_{\varkappa}(z ; h)-K(z ; h)=\mathcal{O}\left(\varkappa^{\infty}\right)=\mathcal{O}\left(h^{\infty}\right), \quad \text { uniformly on } \Gamma_{0} .
$$

Consequently, Lemma 4.2 remains true under the assumptions (2.7) and (2.15). 


\section{Appendix A. Microhyperbolic functions}

In this section, we prove some technical lemmas on the notion of microhyperbolicity used in our proofs.

Lemma A.1. Let $H \in C^{\infty}\left(\mathbb{R}^{2 n} ; \mathcal{H}_{N}\right)$. The following statements are equivalents

(1) $H$ is microhyperbolic at $\rho_{0} \in \mathbb{R}^{2 n}$ in the direction $T \in \mathbb{R}^{2 n}$.

(2) $\left\langle T, \nabla_{\rho} H\left(\rho_{0}\right)\right\rangle_{\mid \operatorname{ker} H\left(\rho_{0}\right)}$ is strictly positive in the sense of hermitian matrices, i.e. there exists $C>0$ such that

$$
\left(\left\langle T, \nabla_{\rho} H\left(\rho_{0}\right)\right\rangle w, w\right) \geq C|w|^{2}, \quad \forall w \in \operatorname{ker} H\left(\rho_{0}\right) .
$$

Proof. Obviously (1) implies (2).

Assume that (2) is satisfied and let us prove (1). Let $w=w_{1}+w_{2} \in \mathbb{C}^{N}$, with $w_{1} \in$ ker $H\left(\rho_{0}\right)$ and $w_{2} \in \operatorname{ker} H\left(\rho_{0}\right)^{\perp}$. We have :

$$
\begin{aligned}
\left(\left\langle T, \nabla_{\rho} H\left(\rho_{0}\right)\right\rangle w, w\right) & =\left(\left\langle T, \nabla_{\rho} H\left(\rho_{0}\right)\right\rangle w_{1}, w_{1}\right)+\left(\left\langle T, \nabla_{\rho} H\left(\rho_{0}\right)\right\rangle w_{2}, w_{2}\right) \\
& +\sum_{i \neq j=1}^{2}\left(\left\langle T, \nabla_{\rho} H\left(\rho_{0}\right)\right\rangle w_{i}, w_{j}\right)=: I_{1}+I_{2}+I_{3} .
\end{aligned}
$$

By hypothesis, $I_{1}$ satisfies

$$
\left|I_{1}\right| \geq C\left|w_{1}\right|^{2}
$$

On the other hand, we have

$$
\left|I_{2}\right| \leq C^{\prime}\left|w_{2}\right|^{2} \quad \text { and } \quad\left|I_{3}\right| \leq C^{\prime \prime}\left|w_{1}\right|\left|w_{2}\right|<\varepsilon C^{\prime \prime}\left|w_{1}\right|^{2}+\frac{C^{\prime \prime}}{\varepsilon}\left|w_{2}\right|^{2},
$$

for $\varepsilon>0$ small enough and $C^{\prime}, C^{\prime \prime}>0$. Putting together (A.2), w.3. we obtain

$$
\left(\left\langle T, \nabla_{\rho} H\left(\rho_{0}\right)\right\rangle w, w\right) \geq \frac{C}{2}|w|^{2}-\mathcal{O}\left(\frac{1}{\varepsilon}\right)\left|w_{2}\right|^{2} .
$$

Now, the fact that $H\left(\rho_{0}\right): \operatorname{ker} H\left(\rho_{0}\right)^{\perp} \rightarrow \operatorname{ker} H\left(\rho_{0}\right)^{\perp}$ is bijective, implies

$$
\left|H\left(\rho_{0}\right) w_{2}\right| \geq \tilde{C}\left|w_{2}\right|, \quad \forall w_{2} \in \operatorname{ker} H\left(\rho_{0}\right)^{\perp} .
$$

Combining this with A.4 , we get

$$
\left(\left\langle T, \nabla_{\rho} H\left(\rho_{0}\right)\right\rangle w, w\right) \geq \frac{C}{2}|w|^{2}-\mathcal{O}\left(\frac{1}{\varepsilon}\right)\left|H\left(\rho_{0}\right) w_{2}\right|^{2},
$$

which together with the fact that $H\left(\rho_{0}\right) w_{2}=H\left(\rho_{0}\right) w$ implies A.1.

Lemma A.2. Let $F \in C^{\infty}\left(\mathbb{R}^{2 n} ; \mathcal{H}_{N-r}\right)$ and $m(0) \in \mathcal{H}_{r}$ invertible, $r \geq 1$. Assume that for $\rho_{0} \in \mathbb{R}^{2 n}$, there exists $T \in \mathbb{R}^{2 n}$ and $C_{0}>0$ such that

$$
\left(\left\langle T, \nabla_{\rho} F\left(\rho_{0}\right)\right\rangle w, w\right) \geq C_{0}|w|^{2}, \quad \forall w \in \mathbb{C}^{N-r} .
$$

Then

(1) $H(\rho)=\left(\begin{array}{cc}F(\rho) & 0 \\ 0 & m(0)\end{array}\right)$ is microhyperbolic at $\rho_{0}$ in the direction $T$.

(2) If A.5) holds at $\rho_{0}=0$ and $M \in C^{\infty}\left(\mathbb{R}^{2 n}, \mathcal{H}_{N}\right)$ with $M(\rho)=\left(\begin{array}{cc}\mathcal{O}\left(|\rho|^{2}\right) & \mathcal{O}(|\rho|) \\ \mathcal{O}(|\rho|) & \mathcal{O}(|\rho|)\end{array}\right)$, then $H+M$ is microhyperbolic near $\rho_{0}=0$ in the direction $T$. 
Proof. Since $\left\langle T, \nabla_{\rho} H\left(\rho_{0}\right)\right\rangle=\left(\begin{array}{cc}\left\langle T, \nabla_{\rho} F\left(\rho_{0}\right)\right\rangle & 0 \\ 0 & 0\end{array}\right)$ and ker $H\left(\rho_{0}\right) \subset \mathbb{C}^{N-r} \times\left\{0_{r}\right\}$, (1) follows immediately from Lemma A.1.

We have

$$
\left\langle T, \nabla_{\rho} H(0)\right\rangle+\left\langle T, \nabla_{\rho} M(0)\right\rangle=\left(\begin{array}{cc}
\left\langle T, \nabla_{\rho} F(0)\right\rangle & \mathcal{O}(1) \\
\mathcal{O}(1) & \mathcal{O}(1)
\end{array}\right)
$$

Therefore

$$
\left(\left\langle T, \nabla_{\rho} H(0)\right\rangle w, w\right)+\left(\left\langle T, \nabla_{\rho} M(0)\right\rangle w, w\right)=\left(\left\langle T, \nabla_{\rho} F(0)\right\rangle w, w\right) \geq C_{0}|w|^{2}, \quad \forall w \in \mathbb{C}^{N-r} .
$$

Since $\operatorname{ker}(H(0)+M(0))=\operatorname{ker}(H(0)) \subset \mathbb{C}^{N-r}$, it follows from lemma A.1 that $H+M$ is microhyperbolic at $\rho_{0}=0$ in the direction $T$. Then, $H+M$ is microhyperbolic near 0 in the direction $T$.

The main result of this appendix is the following.

Theorem A.3. Let $H \in C^{\infty}\left(\mathbb{R}^{2 n} ; \mathcal{H}_{N}\right)$. Assume that $H$ is microhyperbolic near $\rho_{0} \in \mathbb{R}^{2 n}$ in the direction $T$. There exists $\tilde{H} \in C^{\infty}\left(\mathbb{R}^{2 n} ; \mathcal{H}_{N}\right)$ such that $\tilde{H}=H$ near $\rho_{0}$ and $\tilde{H}$ is uniformly microhyperbolic on $\mathbb{R}^{2 n}$ in the direction $T$. Moreover, we can choose $\tilde{H}$ bounded together with all its derivatives, i.e. $\tilde{H} \in S^{0}\left(\mathbb{R}^{2 n} ; \mathcal{H}_{N}\right)$.

Proof. Without any loss of generality, we may assume that $\rho_{0}=0$. We know that there exists $P$ such that

$$
P H(0) P^{-1}=\left(\begin{array}{cc}
0 & 0 \\
0 & m_{22}
\end{array}\right)
$$

where $m_{22}$ is a diagonal and invertible matrix. Replacing $H(\rho)$ by $P H(\rho) P^{-1}$, we may assume that

$$
H(\rho)=\left(\begin{array}{ll}
m_{11}(\rho) & m_{21}(\rho) \\
m_{12}(\rho) & m_{22}(\rho)
\end{array}\right)
$$

with $m_{11}(0)=0, m_{12}(0)=0, m_{21}(0)=0$ and $m_{22}(0)=m_{22}$. Since $H$ is microhyperbolic at 0 in the direction $T$, it follows from Lemma A.1 that

$$
\left\langle\left(\begin{array}{cc}
\left\langle T, \nabla_{\rho} m_{11}(0)\right\rangle & \left\langle T, \nabla_{\rho} m_{21}(0)\right\rangle \\
\left\langle T, \nabla_{\rho} m_{12}(0)\right\rangle & \left\langle T, \nabla_{\rho} m_{22}\right\rangle
\end{array}\right)\left(\begin{array}{c}
w_{1} \\
0
\end{array}\right),\left(\begin{array}{c}
w_{1} \\
0
\end{array}\right)\right\rangle=\left(\left\langle T, \nabla_{\rho} m_{11}(0)\right\rangle w_{1}, w_{1}\right) \geq C\left|w_{1}\right|^{2}
$$

We recall that $\operatorname{ker}(H(0)) \subset\left\{\left(w_{1}, 0\right) ; w_{1} \in \mathbb{C}^{N-r}\right\}$, with $r=\operatorname{dim} \operatorname{Im}\left(m_{22}\right)$ (due to (A.6)).

Set

$$
H_{0}(\rho):=\left(\begin{array}{cc}
\nabla_{\rho} m_{11}(0) \rho & 0 \\
0 & m_{22}
\end{array}\right), \quad \rho \in \mathbb{R}^{2 n} .
$$

It follows from Lemma A.2 and (A.7) that $H_{0}$ is microhyperbolic at every point $\rho \in \mathbb{R}^{2 n}$ in the direction $T$. Let $\chi \in C_{0}^{\infty}\left(\mathbb{R}^{2 n} ; \mathbb{R}\right)$ be such that $\chi(\rho)=1$ for $|\rho| \leq 1$ and $\chi(\rho)=0$ for $|\rho| \geq 2$. For $\delta>0$, set $\chi_{\delta}(\rho)=\chi\left(\frac{\rho}{\delta}\right)$. We define

$$
H_{\delta}(\rho)=\chi\left(\frac{\rho}{\delta}\right)\left(H(\rho)-H_{0}(\rho)\right)+H_{0}(\rho) .
$$

We claim that for $\delta$ small enough, $H_{\delta}$ is microhyperbolic at every point $\rho \in \mathbb{R}^{2 n}$ in the direction $T$. In fact, for $|\rho| \leq \delta, H_{\delta}(\rho)=H(\rho)$ is microhyperbolic at $\rho_{0}=0$ and then at every 
$\rho \in \mathbb{R}^{2 n}$ with $|\rho| \leq \delta$. For $|\rho| \geq 2 \delta, H_{\delta}(\rho)=H_{0}(\rho)$ which is microhyperbolic at every point $\rho \in \mathbb{R}^{2 n}$ in the direction $T$. For $\delta<|\rho|<2 \delta$, we have

$$
H_{\delta}(\rho)=H_{0}(\rho)+\left(\begin{array}{cc}
\mathcal{O}\left(|\rho|^{2}\right) & \mathcal{O}(|\rho|) \\
\mathcal{O}(|\rho|) & \mathcal{O}(|\rho|)
\end{array}\right) .
$$

Thus, Lemma A.2 implies that $H_{\delta}$ is microhyperbolic in the direction $T$ for $\delta$ small enough. Consequently $H_{\delta}$ is microhyperbolic at every point $\rho \in \mathbb{R}^{2 n}$ in the direction $T$. To see that we can choose $\tilde{H} \in S^{0}\left(\mathbb{R}^{2 n} ; \mathcal{H}_{N}\right)$, let $f \in C^{\infty}(\mathbb{R})$ such that $f(t)=t$ for $|t|<1,|f(t)| \geq 1$ on $|t| \geq 1$ and $f(t)$ is constant at $\pm \infty$. Put $\tilde{H}(x)=f\left(H_{\delta}(x)\right)$. By the functional calculus of self-adjoint operator, it is easy to check that $\tilde{H}$ satisfies the desired properties.

Proposition A.4. Let $H \in C^{\infty}\left(\mathbb{R}^{2 n} ; \mathcal{H}_{N}\right), \chi \in C_{0}^{\infty}\left(\mathbb{R}^{2 n}\right)$ and $\tau_{0} \in \mathbb{R}$. Assume that $\tau_{0}-H(\rho)$ is microhyperbolic at every $\rho \in \operatorname{supp} \chi$. Let $G(\rho, z)$ be an $N \times N$ matrix-valued function (not necessarily Hermitian) smooth with respect to $\rho$ and holomorphic with respect to $z$ in a neighborhood of $\tau_{0}$. Set, for $\pm \Im z>0$ respectively,

$$
F_{ \pm}(z)=\int_{\mathbb{R}^{2 n}}(z-H(\rho))^{-1} G(\rho, z)(z-H(\rho))^{-1} \chi(\rho) d \rho .
$$

Then, for real $\tau$ near $\tau_{0}$, the limit $F_{ \pm}(\tau \pm i 0):=\lim _{\varepsilon}{ }_{\searrow} F_{ \pm}(\tau \pm i \varepsilon)$ exists and $\tau \rightarrow F_{ \pm}(\tau \pm i 0)$ is smooth near $\tau_{0}$.

Proof. We consider $F_{+}$. The proof for $F_{-}$is similar. Decomposing $\chi$ into a finite sum of functions $\chi_{i}$ with small support, we may assume using Theorem A.3 that $\tau-H(\rho)$ is microhyperbolic in the direction $T$ at every point $\rho \in \mathbb{R}^{2 n}$ and $\tau$ near $\tau_{0}$. We may also assume that $G, H \in S^{0}\left(\mathbb{R}^{2 n} ; \mathcal{H}_{N}\right)$. Let $\tilde{H}, \tilde{G}$ and $\tilde{\chi}$ be three almost analytic extensions of $H$, $G$ and $\chi$ respectively, which are bounded together with all their derivatives. Put

$$
\tilde{H}(\rho, t):=\tilde{H}(\rho+i t T), \quad \tilde{G}(\rho, t, z):=\tilde{G}(\rho+i t T, z), \quad \tilde{\chi}(\rho, t):=\tilde{\chi}(\rho+i t T), \quad t \in \mathbb{R} .
$$

We assert that for small enough $\Im z \geq 0, t \geq 0$ with $\Im z+t>0$, there exist $C, c>0$ such that

$$
\Im((z-\tilde{H}(\rho, t)) \omega, \omega)+C t|(z-\tilde{H}(\rho, t)) \omega|^{2} \geq c(t+\Im z)|\omega|^{2}, \quad \forall \omega \in \mathbb{C}^{N} .
$$

In fact

$$
((z-\tilde{H}(\rho, t)) \omega, \omega)=((z-H(\rho)) \omega, \omega)-i t\left(\left\langle T, \nabla_{\rho} H(\rho)\right\rangle \omega, \omega\right)+\mathcal{O}\left(t^{2}\right)|\omega|^{2}
$$

and hence the global microhyperbolic condition (see (2.1) ) yields, for some $c, C_{1}, C_{2}>0$,

$$
\begin{gathered}
\Im((z-\tilde{H}(\rho, t)) \omega, \omega) \geq(\Im z+c t)|\omega|^{2}-\mathcal{O}(t)|(\Re z-H(\rho)) \omega|^{2}+\mathcal{O}\left(t^{2}\right)|\omega|^{2} \\
\geq c\left(\Im z+t-C_{1}(\Im z)^{2}-C_{2} t^{2}\right)|\omega|^{2}-\mathcal{O}(t)|(z-\tilde{H}(\rho, t)) \omega|^{2},
\end{gathered}
$$

uniformly on $\{z \in \mathbb{C} ; \Re z \in] \tau_{0}-\eta, \tau_{0}+\eta[, \Im z>0\}$ for small enough $\eta$, and (A.8) follows from this inequality.

Applying Cauchy-Schwarz inequality to the first term of (A.8), we easily obtain

$$
\|z-\tilde{H}(\rho, t)\|_{N \times N}+C t\|z-\tilde{H}(\rho, t)\|_{N \times N}^{2} \geq c(\Im z+t)|\omega|^{2}, \forall \omega \in \mathbb{C}^{N} .
$$

This shows that $(z-\tilde{H}(\rho, t))^{-1}$ exists and

$$
\left\|(z-\tilde{H}(\rho, t))^{-1}\right\|_{N \times N}=\mathcal{O}\left(\frac{1}{t}\right) .
$$

for $t>0, \Im z \geq 0$. 
For simplicity, assume $T=(1,0, \cdots, 0)$. Put $\rho=\left(\rho_{1}, \rho^{\prime}\right)$ and fix $t_{0}>0$. By the Stokes' formula, we have

$$
\begin{aligned}
F_{+}(z) & =\int_{\mathbb{R}^{2 n}}\left(z-\tilde{H}\left(\rho_{1}+i t_{0}, \rho^{\prime}\right)\right)^{-1} \tilde{G}\left(\rho_{1}+i t_{0}, \rho^{\prime}, z\right)\left(z-\tilde{H}\left(\rho_{1}+i t_{0}, \rho^{\prime}\right)\right)^{-1} \tilde{\chi}\left(\rho_{1}+i t_{0}, \rho^{\prime}\right) d \rho \\
& -\iint_{\mathbb{R}^{2 n} \times\left[0, t_{0}\right]} \frac{1}{2}\left(\partial_{\rho_{1}}+i \partial_{t}\right)\left[(z-\tilde{H}(\rho, t))^{-1} \tilde{G}(\rho, z, t)(z-\tilde{H}(\rho, t))^{-1} \tilde{\chi}(\rho, t)\right] d t d \rho .
\end{aligned}
$$

Clearly the first term of the right hand side of the above equality extends to a $C^{\infty}$ function up to $\Im z \geq 0$. One sees that the same is true for the second term by using (A.10) and the fact that $\left(\partial_{\rho_{1}}+i \partial_{t}\right) \tilde{H},\left(\partial_{\rho_{1}}+i \partial_{t}\right) \tilde{G},\left(\partial_{\rho_{1}}+i \partial_{t}\right) \tilde{\chi}$ are all of $\mathcal{O}\left(t^{\infty}\right)$. This ends the proof.

Acknowledgement. This research was initiated when the first and second authors was visiting the Ritsumeikan University in May 2016; the financial support and kind hospitality are gratefully acknowledged. The first author acknowledges support from JSPS KAKENHI Grant number JP16H03944. The third author was partially supported by the JSPS KAKENHI Grant number JP15K04971.

\section{REFERENCES}

[1] M. S. Birman, M. G, Krein, On the theory of wave operators and scattering operators, Dokl. Akad. Nauk SSSR, 144 (1962), 475-478.

[2] M. S. Birman, D. R. Yafaev, On the trace-class method in potential scattering theory, J. Soviet Math, 56, no 2 (1993), 2285-2299.

[3] M. S. Birman, D. R. YAfAev, The spectral shift function. The work of M. G. Krein and its further development, Algebra i Analiz 4, no 5 (1992), 1-44, English trans in St Petersburgh Math J. 4, no 5 (1993).

[4] V. Bruneau, D. Robert, Asymptotics of the scattering phase for the Dirac operator" High energy, semi-classical and non-relativistic limits, Ark. Mat. 37, (199), 1-32.

[5] J. M. Combes, P. Duclos, R. Seiler, The Born-Oppenheimer Approximation, ed. C. G. Velo and A. Wightman, Plenum Press, New York, (1981).

[6] M. Combescure, D. Robert, Coherent states and application in Mathematical Physics, Theoretical and Mathematical Physics (2012).

[7] M. Dimassi, J. Sjöstrand, Spectral asymptotics in the semi-classical limit, London Mathematical Society, Lecture Note Series 268 (1999).

[8] M. Dimassi, S. FujiIE, A time-independent approach for the study of the spectral shift function and an application to Stark Hamiltonians, Comm. in Part. Diff. Equ., vol 40, Issue 10 (2015), 1787-1814.

[9] C. GÉrard, A. Martinez, Principe d'absorption limite pour des opérateurs de Schrödinger à longue portée, C.R. Acad. Sci. 306 (1987), 121-123.

[10] G. A. Hagedorn, Molecular propagation through electron energy level crossing, Mem. Amer. Math. Soc. 111 (1994), no 536.

[11] L. Hörmander, The analysis of linear partial differential operators, vol I-IV, Springer Verlag Berlin Heidelberg New York (1985)

[12] L. Hörmander, Fourier integral operators I, Ada Math. 127 (1971), 79-183.

[13] H. Isozaki, H. Kitada, Modified wave operators with time independent modifiers, J. Math. Phys 7 (1983), $137-143$.

[14] V. IVRII, Microlocal analysis and precise spectral asymptotics, Springer-Verlag, Berlin, (1998).

[15] T. JECKO, Estimation de la résolvante pour une molécule diatomique dans l'approximation de BornOppenheimer, Comm. Math. Phys. 195 (3) (1998), 585-612.

[16] T. Kato, Perturbation theory for linear operators, Springer-Verlag (1995).

[17] M. Klein, A. Martinez, R. Seiler, X. P. Wang, On the Born-Oppenheimer expansion for polyatomic molecules, Comm. Math. Phys. 143 (1992), 607-639. 
[18] A. Khochman, Resonances and spectral shift function for the semi-classical Dirac operator, Rev. Math. Phys., 19 (2007), 1071-115.

Read More: http://www.worldscientific.com/doi/abs/10.1142/S0129055X0700319X?journalCode=rmp

[19] M. G. KREIN, On certain new studies in the perturbation theory for self-adjoint operators, Topics in Differential and Integral Equations and Operators Theory, I Gohberg, Birkhaüser, Basel (1983).

[20] M. G. Krein, On the trace formula in perturbation theory, Mat. Sb, 33 (75) (1953), 597-626 (in russian).

[21] M. G. Krein, V. A. JavrJan, On spectral shift functions arising in perturbation of a positive operator, J. Operator. Th. 6 (1981), 155-191 (in russian).

[22] I. M. Lifshits, On a problem of perturbation theory, Uspekhi Mat. Nauk. 7, no 1, 143 (1952), 171-180.

[23] I. M. Lifshits, Some problems of the dynamic theory nonideal crystal lattices, Nuovo Cimento Suppl. 3 (1956), 716-734.

[24] A. Martinez, T. Ramond, J. Sjöstrand, Resonance for non-analytic potentials, Analysis and PDE, vol 2 (2009) No 1, 29-60.

[25] M. Reed, B. Simon, Methods of Modern Mathematical Physics, Tome II, Fourier Analysis, Selfadjointness, Academic Press (1979).

[26] D. Robert, Autour de l'approximation semi-classique, Progress in Mathematics 68 (1987), Birkhäuser.

[27] D. RoBert, Asymptotique de la phase de diffusion à haute énergie pour des perturbations du second ordre du laplacien, Ann. scient. Ec. Norm. Sup. 4ème série, t.25 (1992), 107-134.

[28] D. Robert, Semiclassical asymptotics for the Spectral Shift Function, Amer. Math. Soc. Trans. 189 (1999).

[29] D. RoBert, Relative time-delay for perturbations of elliptic operators and semiclassical asymptotics, J. Funct. Anal 126 (1994), 36-82.

[30] D. Robert, H. TAmura, Semiclassical bounds for resolvents of Schrödinger operators and asymptotics for scattering phases, Comm. Part. Diff. Equ. 9, no 10, (1984), 1017-1058.

[31] D. Robert, H. Tamura, Semiclassical asymptotics for local spectral densities and time delay problems in scattering processus, J. Funct. Anal. 80 (1) (1988), 124-147.

[32] J. SjÖstrand, M. Zworski, Fractal upper bounds on the density of semiclassical resonances, Duke Math, J. 137 (2007), no 3, 381-459.

[33] X. P. WAng, Time-decay of scattering solutions and resolvent estimates for semiclassical Schrödinger operators, J. Diff. Equations, 71(1988), 348-395.

[34] X. P. Wang, Semiclassical resolvent estimates for N-body Schrödinger operators, J. of Funct. Analysis, 97(1991), 466-483.

[35] X. P. WAng, Time-decay of scattering solutions an classical trajectories. Ann. Inst. H. Poincaré, A, 47(1987), 25-37.

[36] D. YAfAev, Mathematical Scattering Theory, Translation of Mathematical monographs, AMS 105, Providence RI, (1992).

Marouane Assal, Mouez Dimassi, IMB (UMR-CNRS 5251), UNiverSitÉ DE BORDEAUX, 351 COURS DE LA LIBÉRATION, 33405 TALENCE CEDEX, FRANCE

E-mail address: marouane.assal@math.u-bordeaux.fr

E-mail address: mdimassi@u-bordeaux.fr

Setsuro Fujié, RITSUMEIKAN UNIVERSITY, 1-1-1 NOJI-HIGASHI, 525-8577 KUSATSU, JAPAN

E-mail address: fujiie@fc.ritsumei.ac.jp 